\title{
The $\bar{\partial}$-equation on a non-reduced analytic space
}

\author{
Mats Andersson ${ }^{1}$ • Richard Lärkäng1 ${ }^{1}$
}

Received: 14 August 2017 / Revised: 13 March 2018 / Published online: 30 April 2018

(C) The Author(s) 2018

\begin{abstract}
Let $X$ be a, possibly non-reduced, analytic space of pure dimension. We introduce a notion of $\bar{\partial}$-equation on $X$ and prove a Dolbeault-Grothendieck lemma. We obtain fine sheaves $\mathcal{A}_{X}^{q}$ of $(0, q)$-currents, so that the associated Dolbeault complex yields a resolution of the structure sheaf $\mathscr{O}_{X}$. Our construction is based on intrinsic semi-global Koppelman formulas on $X$.
\end{abstract}

Mathematics Subject Classification 32A26 · 32A27 - 32B15 · 32C30

\section{Introduction}

Let $X$ be a smooth complex manifold of dimension $n$ and let $\mathscr{E}_{X}^{0, *}$ denote the sheaf of smooth $(0, *)$-forms. It is well-known that the Dolbeault complex

$$
0 \rightarrow \mathscr{O}_{X} \stackrel{i}{\rightarrow} \mathscr{E}_{X} 0,0 \stackrel{\bar{\partial}}{\rightarrow} \mathscr{E}_{X}^{0,1} \stackrel{\bar{\partial}}{\rightarrow} \cdots \stackrel{\bar{\partial}}{\rightarrow} \mathscr{E}_{X}^{0, n} \rightarrow 0
$$

Communicated by Ngaiming Mok.

The authors were partially supported by grants from the Swedish Research Council.

$凶$ Richard Lärkäng

larkang@chalmers.se

Mats Andersson

matsa@chalmers.se

1 Division of Algebra and Geometry, Department of Mathematical Sciences, Chalmers University of Technology and the University of Gothenburg, 41296 Göteborg, Sweden 
is exact, and hence provides a fine resolution of the structure sheaf $\mathscr{O}_{X}$. If $X$ is a reduced analytic space of pure dimension, then there is still a natural notion of "smooth forms". In fact, assume that $X$ is locally embedded as $i: X \rightarrow \Omega$, where $\Omega$ is a pseudoconvex domain in $\mathbb{C}^{N}$. If $\mathcal{K}$ er $i^{*}$ denotes the subsheaf of all smooth forms $\xi$ in ambient space such that $i^{*} \xi=0$ on the regular part $X_{\text {reg }}$ of $X$, then one defines the sheaf $\mathscr{E}_{X}$ of smooth forms on $X$ simply as

$$
\mathscr{E}_{X}:=\mathscr{E}_{\Omega} / \mathcal{K} \text { er } i^{*}
$$

It is well-known that this definition is independent of the choice of embedding of $X$. Currents on $X$ are defined as the duals of smooth forms with compact support. It is readily seen that the currents $\mu$ on $X$ so defined are in a one-to-one correspondence to the currents $\hat{\mu}=i_{*} \mu$ in ambient space such that $\hat{\mu}$ vanish on $\mathcal{K} e r i_{*}$, see, e.g., [6]. There is an induced $\bar{\partial}$-operator on smooth forms and currents on $X$. In particular, (1.1) is a complex on $X$ but in general it is not exact. In [6], Samuelsson and the first author introduced, by means of intrinsic Koppelman formulas on $X$, fine sheaves $\mathscr{A}_{X}^{*}$ of $(0, *)$-currents that are smooth on $X_{\text {reg }}$ and with mild singularities at the singular part of $X$, such that

$$
0 \rightarrow \mathscr{O}_{X} \stackrel{i}{\rightarrow} \mathscr{A}_{X}^{0} \stackrel{\bar{\partial}}{\rightarrow} \mathscr{A}_{X}^{1} \stackrel{\bar{\partial}}{\rightarrow} \cdots \stackrel{\bar{\partial}}{\rightarrow} \mathscr{A}_{X}^{n} \rightarrow 0
$$

is exact, and thus a fine resolution of the structure sheaf $\mathscr{O}_{X}$. An immediate consequence is the representation

$$
H^{q}\left(X, \mathscr{O}_{X}\right)=\frac{\operatorname{Ker}\left(\mathscr{A}^{0, q}(X) \stackrel{\bar{\partial}}{\rightarrow} \mathscr{A}^{0, q+1}(X)\right)}{\operatorname{Im}\left(\mathscr{A}^{0, q-1}(X) \stackrel{\bar{\partial}}{\rightarrow} \mathscr{A}^{0, q}(X)\right)}, \quad q \geq 1,
$$

of sheaf cohomology, and so (1.3) is a generalization of the classical Dolbeault isomorphism. In special cases more qualitative information of the sheaves $\mathscr{A}_{X}^{q}$ are known, see, e.g., [5,23].

Starting with the influential works $[28,29]$ by Pardon and Stern, there has been a lot of progress recently on the $L^{2}-\bar{\partial}$ theory on non-smooth (reduced) varieties; see, e.g., $[15,27,31]$. The point in these works, contrary to [6], is basically to determine the obstructions to solve $\bar{\partial}$ locally in $L^{2}$. For a more extensive list of references regarding the $\bar{\partial}$-equation on reduced singular varieties, see, e.g., [6].

In [17], a notion of the $\bar{\partial}$-equation on non-reduced local complete intersections was introduced, and which was further studied in [18]. We discuss below how their work relates to ours.

The aim of this paper is to extend the construction in [6] to a non-reduced puredimensional analytic space. The first basic problem is to find appropriate definitions of forms and currents on $X$. Let $X_{\text {reg }}$ be the part of $X$ where the underlying reduced space $Z$ is smooth, and in addition $\mathscr{O}_{X}$ is Cohen-Macaulay. On $X_{\text {reg }}$ the structure sheaf $\mathscr{O}_{X}$ has a structure as a free finitely generated $\mathscr{O}_{Z}$-module. More precisely, assume that we have a local embedding $i: X \rightarrow \Omega \subset \mathbb{C}^{N}$ and coordinates $(z, w)$ in $\Omega$ such that 
$Z=\{w=0\}$. Let $\mathcal{J}$ be the defining ideal sheaf for $X$ on $\Omega$. Then there are monomials $1, w^{\alpha_{1}}, \ldots, w^{\alpha_{\nu-1}}$ such that each $\phi$ in $\mathscr{O}_{\Omega} / \mathcal{J} \simeq \mathscr{O}_{X}$ has a unique representation

$$
\phi=\hat{\phi}_{0} \otimes 1+\hat{\phi}_{1} \otimes w^{\alpha_{1}}+\cdots+\hat{\phi}_{v-1} \otimes w^{\alpha_{v-1}}
$$

where $\hat{\phi}_{j}$ are in $\mathscr{O}_{Z}$. A reasonable notion of a smooth form on $X$ should admit a similar representation on $X_{\text {reg }}$ with smooth forms $\hat{\phi}_{j}$ on $Z$. We first introduce the sheaves $\mathscr{E}_{X}^{0, *}$ of smooth $(0, *)$-forms on $X$. By duality, we then obtain the sheaf $\mathcal{C}_{X}^{n, *}$ of $(n, *)$ currents. We are mainly interested in the subsheaf $\mathcal{P} \mathcal{M}_{X}^{n, *}$ of pseudomeromorphic currents, and especially, the even more restricted sheaf $\mathcal{W}_{X}^{n, *}$ of such currents with the so-called standard extension property, SEP, on $X$. A current with the SEP is, roughly speaking, determined by its restriction to any dense Zariski-open subset.

Of special interest is the sheaf $\omega_{X}^{n} \subset \mathcal{W}_{X}^{n, 0}$ of $\bar{\partial}$-closed pseudomeromorphic $(n, 0)$ currents. In the reduced case this is precisely the sheaf of holomorphic $(n, 0)$-forms in the sense of Barlet-Henkin-Passare, see, e.g., $[12,16]$.

We have no definition of "smooth $(n, *)$-form" on $X$. In order to define $(0, *)$ currents, we use instead the sheaf $\omega_{X}^{n}$ in the following way. Any holomorphic function defines a morphism in $\mathcal{H o m}\left(\omega_{X}^{n}, \omega_{X}^{n}\right)$, and it is a reformulation of a fundamental result of Roos [30], that this morphism is indeed injective, and generically surjective. In the reduced case, multiplication by a current in $\mathcal{W}_{X}^{0, *}$ induces a morphism in $\mathcal{H} \operatorname{Hom}\left(\omega_{X}^{n}, \mathcal{W}_{X}^{n, *}\right)$, and in fact $\mathcal{W}_{X}^{0, *} \rightarrow \mathcal{H} \operatorname{Hom}\left(\omega_{X}^{n}, \mathcal{W}_{X}^{n, *}\right)$ is an isomorphism. In the non-reduced case, we then take this as the definition of $\mathcal{W}_{X}^{0, *}$. It turns out that with this definition, on $X_{\text {reg }}$, any element of $\mathcal{W}_{X}^{0, *}$ admits a unique representation (1.4), where $\hat{\phi}_{j}$ are in $\mathcal{W}_{Z}^{0, *}$, see Sect. 6 below for details.

Given $v, \phi$ in $\mathcal{W}_{X}^{0, *}$ we say that $\bar{\partial} v=\phi$ if $\bar{\partial}(v \wedge h)=\phi \wedge h$ for all $h$ in $\omega_{X}^{n}$. Following [6] we introduce semi-global integral formulas and prove that if $\phi$ is a smooth $\bar{\partial}$-closed $(0, q+1)$-form there is locally a current $v$ in $\mathcal{W}_{X}^{0, q}$ such that $\bar{\partial} v=\phi$. A crucial problem is to verify that the integral operators preserve smoothness on $X_{r e g}$ so that the solution $v$ is indeed smooth on $X_{r e g}$. By an iteration procedure as in [6] we can define sheaves $\mathscr{A}_{X}^{k} \subset \mathcal{W}_{X}^{0, k}$ and obtain our main result in this paper.

Theorem 1.1 Let $X$ be an analytic space of pure dimension $n$. There are sheaves $\mathscr{A}_{X}^{k} \subset \mathcal{W}_{X}^{0, k}$ that are modules over $\mathscr{E}_{X}^{0, *}$, coinciding with $\mathscr{E}_{X}^{0, k}$ on $X_{\text {reg }}$, and such that (1.2) is a resolution of the structure sheaf $\mathscr{O}_{X}$.

The main contribution in this article compared to [6] is the development of a theory for smooth $(0, *)$-forms and various classes of $(n, *)$ - and $(0, *)$-currents in the nonreduced case as is described above. This is done in Sects. $4-8$. The construction of integral operators to provide solutions to $\bar{\partial}$ in Sect. 9 and the construction of the fine resolution of $\mathscr{O}_{X}$ in Sect. 11, which proves Theorem 1.1, are done pretty much in the same way as in [6]. The proof of the smoothness of the solutions of the regular part in Sect. 10 however becomes significantly more involved in the non-reduced case and requires completely new ideas. In Sect. 12 we discuss the relation to the results in $[17,18]$ in case $X$ is a local complete intersection. 


\section{Pseudomeromorphic currents}

Let $s_{1}, \ldots, s_{m}$ be coordinates in $\mathbb{C}^{m}$, let $\alpha$ be a smooth form with compact support, and let $a_{1}, \ldots, a_{r}$ be positive integers, $0 \leq \ell \leq r \leq m$. Then

$$
\bar{\partial} \frac{1}{s_{1}^{a_{1}}} \wedge \cdots \wedge \bar{\partial} \frac{1}{s_{\ell}^{a_{\ell}}} \wedge \frac{\alpha}{s_{\ell+1}^{a_{\ell+1}} \cdots s_{r}^{a_{r}}}
$$

is a well-defined current that we call an elementary (pseudomeromorphic) current. Let $Z$ be a reduced space of pure dimension. A current $\tau$ is pseudomeromorphic on $Z$ if, locally, it is the push-forward of a finite sum of elementary pseudomeromorphic currents under a sequence of modifications, simple projections, and open inclusions. The pseudomeromorphic currents define an analytic sheaf $\mathcal{P} \mathcal{M}_{Z}$ on $Z$. This sheaf was introduced in [8] and somewhat extended in [6]. If nothing else is explicitly stated, proofs of the properties listed below can be found in, e.g., [6].

If $\tau$ is pseudomeromorphic and has support on an analytic subset $V$, and $h$ is a holomorphic function that vanishes on $V$, then $\bar{h} \tau=0$ and $d \bar{h} \wedge \tau=0$.

Given a pseudomeromorphic current $\tau$ and a subvariety $V$ of some open subset $\mathcal{U} \subset Z$, the natural restriction to the open $\operatorname{set} \mathcal{U} \backslash V$ of $\tau$ has a natural extension to a pseudomeromorphic current on $\mathcal{U}$ that we denote by $\mathbf{1}_{\mathcal{U} \backslash V} \tau$. Throughout this paper we let $\chi$ denote a smooth function on $[0, \infty)$ that is 0 in a neighborhood of 0 and 1 in a neighborhood of $\infty$. If $h$ is a holomorphic tuple whose common zero set is $V$, then

$$
\mathbf{1}_{\mathcal{U} \backslash V} \tau=\lim _{\epsilon \rightarrow 0^{+}} \chi\left(|h|^{2} / \epsilon\right) \tau
$$

Notice that $\mathbf{1}_{V} \tau:=\left(1-\mathbf{1}_{\mathcal{U} \backslash V}\right) \tau$ is also pseudomeromorphic and has support on $V$. If $W$ is another analytic set, then

$$
\mathbf{1}_{V} \mathbf{1}_{W} \tau=\mathbf{1}_{V \cap W} \tau
$$

This action of $\mathbf{1}_{V}$ on the sheaf of pseudomeromorphic currents is a basic tool. In fact one can extend this calculus to all constructible sets so that (2.2) holds, see [8]. One readily checks that if $\xi$ is a smooth form, then

$$
\mathbf{1}_{V}(\xi \wedge \tau)=\xi \wedge \mathbf{1}_{V} \tau
$$

If $f: Z^{\prime} \rightarrow Z$ is a modification and $\tau$ is in $\mathcal{P} \mathcal{M}_{Z^{\prime}}$ then $f_{*} \tau$ is in $\mathcal{P} \mathcal{M}_{Z}$. The same holds if $f$ is a simple projection and $\tau$ has compact support in the fiber direction. In any case we have

$$
\mathbf{1}_{V} f_{*} \tau=f_{*}\left(\mathbf{1}_{f^{-1} V} \tau\right)
$$

It is not hard to check that if $\tau$ is in $\mathcal{P} \mathcal{M}_{Z}$ and $\tau^{\prime}$ is in $\mathcal{P} \mathcal{M}_{Z^{\prime}}$, then $\tau \otimes \tau^{\prime}$ is in $\mathcal{P} \mathcal{M}_{Z \times Z^{\prime}}$, see, e.g., [4, Lemma 3.3]. If $V \subset \mathcal{U} \subset Z$ and $V^{\prime} \subset \mathcal{U}^{\prime} \subset Z^{\prime}$, then

$$
\left(\mathbf{1}_{V} \tau\right) \otimes \mathbf{1}_{V^{\prime}} \tau^{\prime}=\mathbf{1}_{V \times V^{\prime}}\left(\tau \otimes \tau^{\prime}\right)
$$


Another basic tool is the dimension principle, that states that if $\tau$ is a pseudomeromorphic $(*, p)$-current with support on an analytic set with codimension larger than $p$, then $\tau$ must vanish.

A pseudomeromorphic current $\tau$ on $Z$ has the standard extension property, SEP, if $\mathbf{1}_{V} \tau=0$ for each germ $V$ of an analytic set with positive codimension on $Z$. The set $\mathcal{W}_{Z}$ of all pseudomeromorphic currents on $Z$ with the SEP is a subsheaf of $\mathcal{P} \mathcal{M}_{Z}$. By (2.3), $\mathcal{W}_{Z}$ is closed under multiplication by smooth forms.

Let $f$ be a holomorphic function (or a holomorphic section of a Hermitian line bundle), not vanishing identically on any irreducible component of $Z$. Then $1 / f$, a priori defined outside of $\{f=0\}$, has an extension as a pseudomeromorphic current, the principal value current, still denoted by $1 / f$, such that $\mathbf{1}_{\{f=0\}}(1 / f)=0$. The current $1 / f$ has the SEP and

$$
\frac{1}{f}=\lim _{\epsilon \rightarrow 0^{+}} \chi\left(|f|^{2} / \epsilon\right) \frac{1}{f} .
$$

We say that a current $a$ on $Z$ is almost semi-meromorphic if there is a modification $\pi: Z^{\prime} \rightarrow Z$, a holomorphic section $f$ of a line bundle $L \rightarrow Z^{\prime}$ and a smooth form $\gamma$ with values in $L$ such that $a=\pi_{*}(\gamma / f)$, cf., [10, Section 4]. If $a$ is almost semi-meromorphic, then it is clearly pseudomeromorphic. Moreover, it is smooth outside an analytic set $V \subset Z$ of positive codimension, $a$ is in $\mathcal{W}_{Z}$, and in particular, $a=\lim _{\epsilon \rightarrow 0^{+}} \chi(|h| / \epsilon) a$ if $h$ is a holomorphic tuple that cuts out (an analytic set of positive codimension that contains) $V$. The Zariski singular support of $a$ is the Zariski closure of the set where $a$ is not smooth.

One can multiply pseudomeromorphic currents by almost semi-meromorphic currents; and this fact will be crucial in defining $\mathcal{W}_{X}^{0, *}$, when $X$ is non-reduced. Notice that if $a$ is almost semi-meromorphic in $Z$ then it also is in any open $\mathcal{U} \subset Z$.

Proposition 2.1 ([10, Theorem 4.8, Proposition 4.9]) Let $Z$ be a reduced space, assume that $a$ is an almost semi-meromorphic current in $Z$, and let $V$ be the Zariski singular support of $a$.

(i) If $\tau$ is a pseudomeromorphic current in $\mathcal{U} \subset Z$, then there is a unique pseudomeromorphic current $a \wedge \tau$ in $\mathcal{U}$ that coincides with (the naturally defined current) $a \wedge \tau$ in $\mathcal{U} \backslash V$ and such that $\mathbf{1}_{V}(a \wedge \tau)=0$.

(ii) If $W \subset \mathcal{U}$ is any analytic subset, then

$$
\mathbf{1}_{W}(a \wedge \tau)=a \wedge \mathbf{1}_{W} \tau
$$

Notice that if $h$ is a tuple that cuts out $V$, then in view of (2.1),

$$
a \wedge \tau=\lim _{\epsilon \rightarrow 0^{+}} \chi\left(|h|^{2} / \epsilon\right) a \wedge \tau .
$$

It follows that if $\xi$ is a smooth form, then

$$
\xi \wedge(a \wedge \tau)=(-1)^{\operatorname{deg} \xi \operatorname{deg} a} a \wedge(\xi \wedge \tau) .
$$


For future reference we will need the following result.

Proposition 2.2 Let $Z$ be a reduced space. Then $\mathcal{P} \mathcal{M}_{Z}=\mathcal{W}_{Z}+\bar{\partial} \mathcal{W}_{Z}$.

Proof First assume that $Z$ is smooth. Since $\mathcal{W}_{Z}$ is closed under multiplication by smooth forms, so is $\mathcal{W}_{Z}+\bar{\partial} \mathcal{W}_{Z}$. The statement that $\mathcal{P} \mathcal{M}_{Z}=\mathcal{W}_{Z}+\bar{\partial} \mathcal{W}_{Z}$ is local, and since both sides are closed under multiplication by cutoff functions, we may consider a pseudomeromorphic current $\mu$ with compact support in $\mathbb{C}^{n}$. If $\mu$ has bidegree $(*, 0)$, then it is in $\mathcal{W}_{Z}$ in view of the dimension principle. Thus we assume that $\mu$ has bidegree $(*, q)$ with $q \geq 1$. Let

$$
K \mu(z)=\int_{\zeta} k(\zeta, z) \wedge \mu(\zeta),
$$

where $k$ is the Bochner-Martinelli kernel. Here (2.9) means that $K \mu=p_{*}(k \wedge \mu \otimes 1)$, where $p$ is the projection $\mathbb{C}_{\zeta}^{n} \times \mathbb{C}_{z}^{n} \rightarrow \mathbb{C}_{z}^{n}, \quad(\zeta, z) \mapsto z$. Recall that we have the Koppelman formula $\mu=\bar{\partial} K \mu+K(\bar{\partial} \mu)$. It is thus enough to see that $K \mu$ is in $\mathcal{W}_{Z}$ if $\mu$ is pseudomeromorphic. Let $\chi_{\epsilon}=\chi\left(|\zeta-z|^{2} / \epsilon\right)$. It is easy to see, by a blowup of $\mathbb{C}^{n} \times \mathbb{C}^{n}$ along the diagonal, that $k$ is almost semi-meromorphic on $\mathbb{C}^{n} \times \mathbb{C}^{n}$. Thus, by (2.7), $\chi_{\epsilon} k \wedge(\mu \otimes 1) \rightarrow k \wedge(\mu \otimes 1)$. In view of Proposition 2.1 it follows that $k \wedge(\mu \otimes 1)$ is pseudomeromorphic. Finally, if $W$ is a germ of a subvariety of $\mathbb{C}^{n}$ of positive codimension, then by (2.4) and (2.5),

$$
\begin{aligned}
\mathbf{1}_{W} p_{*}(k \wedge \mu \otimes 1) & =\lim _{\epsilon \rightarrow 0^{+}} p_{*}\left(\mathbf{1}_{\mathbb{C}^{n} \times W}\left(\chi_{\epsilon} k \wedge(\mu \otimes 1)\right)\right) \\
& =\lim _{\epsilon \rightarrow 0^{+}} p_{*}\left(\chi_{\epsilon} k \wedge\left(\mathbf{1}_{\mathbb{C}^{n} \times W} \mu \otimes 1\right)\right) \\
& =\lim _{\epsilon \rightarrow 0^{+}} p_{*}\left(\chi_{\epsilon} k \wedge\left(\mathbf{1}_{\mathbb{C}^{n}} \mu \otimes \mathbf{1}_{W} 1\right)\right)=0,
\end{aligned}
$$

since $\mathbf{1}_{W} 1=0$. Thus $K \mu$ is in $\mathcal{W}_{Z}$.

If $Z$ is not smooth, then we take a smooth modification $\pi: Z^{\prime} \rightarrow Z$. For any $\mu$ in $\mathcal{P} \mathcal{M}_{Z}$ there is some $\mu^{\prime}$ in $\mathcal{P} \mathcal{M}_{Z^{\prime}}$ such that $\pi_{*} \mu^{\prime}=\mu$, see [4, Proposition 1.2]. Since $\mu^{\prime}=\tau+\bar{\partial} u$ with $\tau, u$ in $\mathcal{W}_{Z^{\prime}}$, we have that $\mu=\pi_{*} \tau+\bar{\partial} \pi_{*} u$.

\subsection{Pseudomeromorphic currents with support on a subvariety}

Let $\Omega$ be an open set in $\mathbb{C}^{N}$ and let $Z$ be a (reduced) subvariety of pure dimension $n$. Let $\mathcal{P} \mathcal{M}_{\Omega}^{Z}$ denote the sheaf of pseudomeromorphic currents $\tau$ on $\Omega$ with support on $Z$, and let $\mathcal{W}_{\Omega}^{Z}$ denote the subsheaf of $\mathcal{P} \mathcal{M}_{\Omega}^{Z}$ of currents of bidegree $(N, *)$ with the SEP with respect to $Z$, i.e., such that $\mathbf{1}_{W} \tau=0$ for all germs $W$ of subvarieties of $Z$ of positive codimension. The sheaf $\mathcal{C H}_{\Omega}^{Z}$ of Coleff-Herrera currents on $Z$ is the subsheaf of $\mathcal{W}_{\Omega}^{Z}$ of $\bar{\partial}$-closed $(N, p)$-currents, where $p=N-n$.

Remark 2.3 In $[3,6] \mathcal{C H}_{Z}^{\Omega}$ denotes the sheaf of pseudomeromorphic $(0, p)$-currents with support on $Z$ and the SEP with respect to $Z$. If this sheaf is tensored by the canonical bundle $K_{\Omega}$ we get the sheaf $\mathcal{C} \mathcal{H}_{\Omega}^{Z}$ in this paper. Locally these sheaves are thus isomorphic via the mapping $\mu \mapsto \mu \wedge \alpha$, where $\alpha$ is a non-vanishing holomorphic $(N, 0)$-form. 
We have the following direct consequence of Proposition 2.1.

Proposition 2.4 Let $Z \subset \Omega$ be a subvariety of pure dimension, let a be almost semimeromorphic in $\Omega$, and assume that it is smooth generically on $Z$. If $\tau$ is in $\mathcal{W}_{\Omega}^{Z}$, then $a \wedge \tau$ is in $\mathcal{W}_{\Omega}^{Z}$ as well.

Assume that we have local coordinates $(z, w) \in \mathbb{C}^{n} \times \mathbb{C}^{p}$ in $\Omega$ such that $Z=\{w=$ $0\}$. We will use the short-hand notation

$$
\bar{\partial} \frac{d w}{w^{\gamma+1}}:=\bar{\partial} \frac{d w_{1}}{w_{1}^{\gamma_{1}+1}} \wedge \cdots \wedge \bar{\partial} \frac{d w_{p}}{w_{p}^{\gamma_{p}+1}}
$$

for multiindices $\gamma=\left(\gamma_{1}, \ldots, \gamma_{p}\right)$ with $\gamma_{j} \geq 0$, and let $\gamma !:=\gamma_{1} ! \cdots \gamma_{p} !$. Notice that

$$
\frac{1}{(2 \pi i)^{p}} \bar{\partial} \frac{d w}{w^{\gamma+1}} \cdot \xi=\frac{1}{\gamma !} \int_{z} \frac{\partial^{\gamma} \xi}{\partial w^{\gamma}}(z, 0)
$$

for test forms $\xi$. If $\tau$ is in $\mathcal{W}_{Z}$, then it follows by (2.5) and the fact that $\operatorname{supp} \bar{\partial}\left(1 / w^{\gamma+\mathbf{1}}\right)=\{w=0\}$ that $\tau \otimes \bar{\partial}\left(1 / w^{\gamma+\mathbf{1}}\right)$ is in $\mathcal{W}_{\Omega}^{Z}$. We have the following local structure result, see [11, Proposition 4.1 and (4.3)] and [10, Theorem 3.5].

Proposition 2.5 Assume that we have local coordinates $(z, w)$ such that $Z=\{w=$ $0\}$. Then $\tau$ in $\mathcal{W}_{\Omega}^{Z}$ has a unique representation as a finite sum

$$
\tau=\sum_{\gamma} \tau_{\gamma} \wedge d z \otimes \bar{\partial} \frac{d w}{w^{\gamma+1}}, \quad \tau_{\gamma} \in \mathcal{W}_{Z}^{0, *},
$$

where $d z:=d z_{1} \wedge \cdots \wedge d z_{n}$. If $\pi$ is the projection $(z, w) \mapsto z$, then

$$
\tau_{\gamma} \wedge d z=(2 \pi i)^{-p_{\pi}} \pi_{*}\left(w^{\gamma} \tau\right)
$$

If in addition $\bar{\partial} \tau$ is in $\mathcal{W}_{\Omega}^{Z}$ then its coefficients in the expansion (2.11) are $\bar{\partial} \tau_{\gamma}$, cf., (2.12). In particular, $\bar{\partial} \tau=0$ if and only if $\bar{\partial} \tau_{\gamma}=0$ for all $\gamma$.

Let us now consider the pairing between $\mathcal{W}_{\Omega}^{Z}$ and germs $\phi$ at $Z$ of smooth $(0, *)$ forms. We assume that $Z$ is smooth and that we have coordinates $(z, w)$ as before, that $\tau$ is in $\mathcal{W}_{\Omega}^{Z}$, and that (2.11) holds. Moreover, we assume that $\phi$ is a smooth $(0, *)$-form in a neighborhood of $Z$ in $\Omega$. For any positive integer $M$ we have the expansion

$$
\phi=\sum_{|\alpha|<M} \phi_{\alpha}(z) \otimes w^{\alpha}+\mathscr{O}\left(|w|^{M}\right)+\mathscr{O}(\bar{w}, d \bar{w})
$$

where

$$
\phi_{\alpha}(z)=\frac{1}{\alpha !} \frac{\partial \phi}{\partial w^{\alpha}}(z, 0)
$$


and $\mathscr{O}(\bar{w}, d \bar{w})$ denotes a sum of terms, each of which contains a factor $\bar{w}_{j}$ or $d \bar{w}_{j}$ for some $j$. If $M$ in (2.13) is chosen so that $\mathscr{O}\left(|w|^{M}\right) \tau=0$, then

$$
\phi \wedge \tau=\sum_{\alpha \leq \gamma} \phi_{\alpha} \wedge \tau_{\gamma} \wedge d z \otimes \bar{\partial} \frac{d w}{w^{\gamma-\alpha+1}},
$$

i.e.,

$$
\phi \wedge \tau=\sum_{\ell \geq 0} \sum_{\gamma \geq 0} \phi_{\gamma} \wedge \tau_{\ell+\gamma} \wedge d z \otimes \bar{\partial} \frac{d w}{w^{\ell+\mathbf{1}}} .
$$

Thus $\phi \wedge \tau=0$ if and only if $\sum_{\gamma \geq 0} \phi_{\gamma} \wedge \tau_{\ell+\gamma}=0$ for all $\ell$ (which is a finite number of conditions!).

\subsection{Intrinsic pseudomeromorphic currents on a reduced subvariety}

Currents on a reduced analytic space $Z$ are defined as the dual of the sheaf of test forms. If $i: Z \rightarrow Y$ is an embedding of a reduced space $Z$ into a smooth manifold $Y$, then the push-forward mapping $\tau \mapsto i_{*} \tau$ gives an isomorphism between currents $\tau$ on $Z$ and currents $\mu$ on $Y$ such that $\xi \wedge \mu=0$ for all $\xi$ in $\mathscr{E}_{Y}$ such that $i^{*} \xi=0$.

When defining pseudomeromorphic currents in the non-reduced case it is desirable that it coincides with the previous definition in case $Z$ is reduced. From [4, Theorem 1.1] we have the following description of pseudomeromophicity from the point of view of an ambient smooth space.

Proposition 2.6 Assume that we have an embedding $i: Z \rightarrow Y$ of a reduced space $Z$ into a smooth manifold $Y$.

(i) If $\tau$ is in $\mathcal{P} \mathcal{M}_{Z}$, then $i_{*} \tau$ is in $\mathcal{P} \mathcal{M}_{Y}$.

(ii) If $\tau$ is a current on $Z$ such that $i_{*} \tau$ is in $\mathcal{P} \mathcal{M}_{Y}$ and $\mathbf{1}_{Z_{\text {sing }}}\left(i_{*} \tau\right)=0$, then $\tau$ is in $\mathcal{P} \mathcal{M}_{Z}$.

Since $i_{*}\left(i^{*} \chi\left(|h|^{2} / \epsilon\right) \tau\right)=\chi\left(|h|^{2} / \epsilon\right) i_{*} \tau$ for any current $\tau$ on $Z$, we get by (2.1) that for a subvariety $V \subset \mathcal{U} \subset Z$,

$$
\mathbf{1}_{V}\left(i_{*} \tau\right)=i_{*}\left(\mathbf{1}_{V} \tau\right)
$$

i.e., (2.4) holds also for an embedding $i: Z \rightarrow Y$. The condition $\mathbf{1}_{Z_{\text {sing }}}\left(i_{*} \tau\right)=0$ in (ii) is fulfilled if $i_{*} \tau$ has the SEP with respect to $Z$.

Corollary 2.7 We have the isomorphism

$$
i_{*}: \mathcal{W}_{Z}^{n, *} \rightarrow \mathcal{H} \operatorname{om}\left(\mathscr{O}_{\Omega} / \mathcal{J}, \mathcal{W}_{\Omega}^{Z}\right)
$$

where $\mathcal{J}$ is the ideal defining $Z$ in $\Omega$.

Notice that $\mathcal{H} \operatorname{om}\left(\mathscr{O}_{\Omega} / \mathcal{J}, \mathcal{W}_{\Omega}^{Z}\right)$ is precisely the sheaf of $\mu$ in $\mathcal{W}_{\Omega}^{Z}$ such that $\mathcal{J} \mu=0$. 
Proof The map $i_{*}$ is injective, since it is injective on any currents, and it maps into $\mathcal{H o m}\left(\mathscr{O}_{\Omega} / \mathcal{J}, \mathcal{W}_{\Omega}^{Z}\right)$ by $(2.15)$.

To see that $i_{*}$ is surjective, we take a $\mu$ in $\mathcal{H o m}\left(\mathscr{O}_{\Omega} / \mathcal{J}, \mathcal{W}_{\Omega}^{Z}\right)$. We assume first that

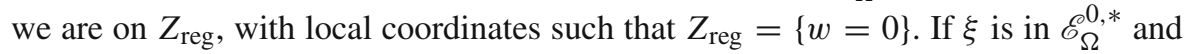
$i^{*} \xi=0$, then $\xi$ is a sum of forms with a factor $d \bar{w}_{j}, w_{j}$ or $\bar{w}_{j}$. Since $w_{j} \in \mathcal{J}, w_{j}$ annihilates $\mu$ by assumption, and since $w_{j}$ vanishes on the support of $\mu, \bar{w}_{j}$ and $d \bar{w}_{j}$ annihilate $\mu$ since $\mu$ is pseudomeromorphic. Thus, $\mu . \xi=0$, so $\mu=i_{*} \tau$ for some current $\tau$ on $Z$. By Proposition 2.6 (ii), $\tau$ is pseudomeromorphic, and by (2.15), has the SEP, i.e., $\tau$ is in $\mathcal{W}_{Z}^{n, *}$.

Remark 2.8 We do not know whether $i_{*} \tau \in \mathcal{P} \mathcal{M}_{\Omega}^{Z}$ implies that $\tau \in \mathcal{P} \mathcal{M}_{Z}$.

By [11, Proposition 3.12 and Theorem 3.14], we get

Proposition 2.9 Let $\varphi$ and $\phi_{1}, \ldots, \phi_{m}$ be currents in $\mathcal{W}_{Z}$. If $\varphi=0$ on the set on $Z_{\text {reg }}$ where $\phi_{1}, \ldots, \phi_{m}$ are smooth, then $\varphi=0$.

\section{Local embeddings of a non-reduced analytic space}

Let $X$ be an analytic space of pure dimension $n$ with structure sheaf $\mathscr{O}_{X}$ and let $Z=X_{\text {red }}$ be the underlying reduced analytic space. For any point $x \in X$ there is, by definition, an open set $\Omega \subset \mathbb{C}^{N}$ and an ideal sheaf $\mathcal{J} \subset \mathscr{O}_{\Omega}$ of pure dimension $n$ with zero set $Z$ such that $\mathscr{O}_{X}$ is isomorphic to $\mathscr{O}_{\Omega} / \mathcal{J}$, and all associated primes of $\mathcal{J}$ at any point have dimension $n$. We say that we have a local embedding $i: X \rightarrow \Omega \subset \mathbb{C}^{N}$ at $x$. There is a minimal such $N$, called the Zariski embedding dimension $\hat{N}$ of $X$ at $x$, and the associated embedding is said to be minimal. Any two minimal embeddings are identical up to a biholomorphism, and any embedding $i: X \rightarrow \Omega$ has locally at $x$ the form

$$
X \stackrel{j}{\rightarrow} \widehat{\Omega} \stackrel{\iota}{\rightarrow} \Omega:=\widehat{\Omega} \times \mathcal{U}, \quad i=\iota \circ j
$$

where $j$ is minimal, $\mathcal{U}$ is an open subset of $\mathbb{C}_{w}^{m}, m=N-\hat{N}$, and the ideal in $\Omega$ is $\mathcal{J}=\widehat{\mathcal{J}} \otimes 1+\left(w_{1}, \ldots, w_{m}\right)$. Notice that we then also have embeddings $Z \rightarrow \widehat{\Omega} \rightarrow \Omega$; however, the first one is in general not minimal.

Now consider a fixed local embedding $i: X \rightarrow \Omega \subset \mathbb{C}^{N}$, assume that $Z$ is smooth, and let $(z, w)$ be coordinates in $\Omega$ such that $Z=\{w=0\}$. We can identify $\mathscr{O}_{Z}$ with holomorphic functions of $z$, and we can define an injection

$$
\mathscr{O}_{Z} \rightarrow \mathscr{O}_{X}, \quad \phi(z) \mapsto \tilde{\phi}(z, w)=\phi(z)
$$

In this way $\mathscr{O}_{X}$ becomes an $\mathscr{O}_{Z}$-module, which however depends on the choice of coordinates.

Proposition 3.1 Assume that $Z$ is smooth. Let $\mathscr{O}_{X}$ have the $\mathscr{O}_{Z}$-module structure from a choice of local coordinates as above. Then $\mathscr{O}_{X}$ is a coherent $\mathscr{O}_{Z}$-module, and $\mathscr{O}_{X}$ is a free $\mathscr{O}_{Z}$-module at $x$ if and only if $\mathscr{O}_{X}$ is Cohen-Macaulay at $x$. 
Recall that $f_{1}, \ldots, f_{m} \in R$ is a regular sequence on the $R$-module $M$ if $f_{i}$ is a non zero-divisor on $M /\left(f_{1}, \ldots, f_{i-1}\right)$ for $i=1, \ldots, m$, and $\left(f_{1}, \ldots, f_{m}\right) M \neq M$. If $R$ is a local ring, then $\operatorname{depth}_{R} M$ is the maximal length $d$ of a regular sequence $f_{1}, \ldots, f_{d}$ such that $f_{1}, \ldots, f_{d}$ are contained in the maximal ideal $\mathfrak{m}$; furthermore, $M$ is CohenMacaulay if $\operatorname{depth}_{R} M=\operatorname{dim}_{R} M$, where $\operatorname{dim}_{R} M=\operatorname{dim}_{R}\left(R / \operatorname{ann}{ }_{R} M\right)$. If $R$ is Cohen-Macaulay, and $M$ has a finite free resolution over $R$, then the AuslanderBuchsbaum formula, [14, Theorem 19.9], gives that

$$
\operatorname{depth}_{R} M+\operatorname{pd}_{R} M=\operatorname{dim}_{R} R
$$

where $\operatorname{pd}_{R} M$ is the length of a minimal free resolution of $M$ over $R$. In this case, $M$ is Cohen-Macaulay as an $R$-module if and only if $M$ has a free resolution over $R$ of length $\operatorname{codim} M$.

Remark 3.2 Notice that if we have a local embedding $i: X \rightarrow \Omega$ as above, then the depth and dimension of $\mathscr{O}_{X, x}=\mathscr{O}_{\Omega, x} / \mathcal{J}$ as an $\mathscr{O}_{\Omega, x}$-module coincide with the depth and dimension of $\mathscr{O}_{X, x}$ as an $\mathscr{O}_{X, x}$-module. Thus $\mathscr{O}_{X, x}$ is Cohen-Macaulay as an $\mathscr{O}_{X, x}$-module if and only if it is Cohen-Macaulay as an $\mathscr{O}_{\Omega, x}$-module, and this holds in turn if and only if $\mathscr{O}_{\Omega, x} / \mathcal{J}$ has a free resolution of length $N-n$.

Proof of Proposition 3.1 By the Nullstellensatz there is an $M$ such that $w^{\alpha}$ is in $\mathcal{J}$ in some neighborhood of $x$ if $|\alpha|=M$. Let $\mathcal{M} \subset \mathscr{O}_{\Omega}$ be the ideal generated by $\left\{w^{\alpha} ;|\alpha|=M\right\}$. Then $\mathcal{M}^{\prime}=\mathscr{O}_{\Omega} / \mathcal{M}$ is a free, finitely generated $\mathscr{O}_{Z}$-module. Thus, $\mathscr{O}_{\Omega} / \mathcal{J} \simeq \mathcal{M}^{\prime} / \mathcal{J} \mathcal{M}^{\prime}$ is a coherent $\mathscr{O}_{Z}$-module, which we note is generated by the finite set of monomials $w^{\alpha}$ such that $|\alpha|<M$.

We shall now show that

$$
\operatorname{depth}_{\mathscr{O}_{X, x}} \mathscr{O}_{X, x}=\operatorname{depth}_{\mathscr{O}_{Z, x}} \mathscr{O}_{X, x}
$$

and

$$
\operatorname{dim}_{\mathscr{O}_{X, x}} \mathscr{O}_{X, x}=\operatorname{dim}_{\mathscr{O}_{Z, x}} \mathscr{O}_{X, x} .
$$

We claim that a sequence $f_{1}, \ldots, f_{m}$ in $\mathscr{O}_{X, x}$ is regular (on $\mathscr{O}_{X, x}$ ) if and only if $\tilde{f}_{1}, \ldots, \tilde{f}_{m} \in \mathscr{O}_{Z, x}$ is regular on $\mathscr{O}_{X, x}$, where $\tilde{f}_{j}(z)=f_{j}(z, 0)$. In fact, since $\mathscr{O}_{X, x}$ has pure dimension, a function $g \in \mathscr{O}_{X, x}=\mathscr{O}_{\Omega, x} / \mathcal{J}$ is a non zero-divisor if and only if $g$ is generically non-vanishing on each irreducible component of $Z(\mathcal{J})$. Thus $f_{1}$ is a non zero-divisor if and only if $\tilde{f}_{1}$ is. If it is, then $\mathscr{O}_{X, x} /\left(f_{1}\right)=\mathscr{O}_{\Omega, x} /\left(\mathcal{J}+\left(f_{1}\right)\right)$ again has pure dimension. Thus the claim follows by induction, and the fact that $Z\left(\mathcal{J}+\left(f_{1}, \ldots, f_{k}\right)\right)=Z\left(\mathcal{J}+\left(\tilde{f}_{1}, \ldots, \tilde{f}_{k}\right)\right)$. The claim immediately implies (3.3).

To see (3.4), we note first that $\operatorname{dim}_{\mathscr{O}_{X, x}} \mathscr{O}_{X, x}$ is just the usual (geometric) dimension of $X$ or $Z$, i.e., in this case, $n$. Now, ann $\mathscr{O}_{Z, x} \mathscr{O}_{X, x}=\{0\}$, so $\operatorname{dim}_{\mathscr{O}_{Z, x}} \mathscr{O}_{X, x}=$ $\operatorname{dim}_{\mathscr{O}_{Z, x}} \mathscr{O}_{Z, x} /\left(\operatorname{ann} \mathscr{O}_{Z, x} \mathscr{O}_{X, x}\right)=\operatorname{dim}_{\mathscr{O}_{Z, x}} \mathscr{O}_{Z, x}=n$.

From (3.3) and (3.4) we conclude that $\mathscr{O}_{X, x}$ is Cohen-Macaulay as an $\mathscr{O}_{Z, x}$-module if and only if it is Cohen-Macaulay (as an $\mathscr{O}_{X, x}$-module). Hence, by (3.2), with $R=\mathscr{O}_{Z, x}$ and $M=\mathscr{O}_{X, x}$,

$$
\operatorname{depth}_{\mathscr{O}_{Z, x}} \mathscr{O}_{X, x}+\operatorname{pd}_{\mathscr{O}_{Z, x}} \mathscr{O}_{X, x}=n,
$$


so $\mathscr{O}_{X, x}$ is Cohen-Macaulay as an $\mathscr{O}_{Z, x}$-module if and only if $\operatorname{pd}_{\mathscr{O}_{Z, x}} \mathscr{O}_{X, x}=0$, that is, if and only if $\mathscr{O}_{X, x}$ is a free $\mathscr{O}_{Z, x}$-module.

In the proof above, we saw that $\mathscr{O}_{X}$ is generated (locally) as an $\mathscr{O}_{Z}$-module by all monomials $w^{\alpha}$ with $|\alpha| \leq M$ for some $M$.

Corollary 3.3 Assume that $1, w^{\alpha_{1}}, \ldots, w^{\alpha_{\nu-1}}$ is a minimal set of generators at a given point $x$ (clearly 1 must be among the generators!). Then we have a unique representation (1.4) for each $\phi \in \mathscr{O}_{X, x}$ if and only if $\mathscr{O}_{X, x}$ is Cohen-Macaulay.

By coherence it follows that if $\mathscr{O}_{X, x}$ is free as an $\mathscr{O}_{Z, x}$-module, then $\mathscr{O}_{Z, x^{\prime}}$ is free as an $\mathscr{O}_{Z, x^{\prime}}$-module for all $x^{\prime}$ in a neighborhood of $x$, and $1, w^{\alpha_{1}}, \ldots, w^{\alpha_{\nu-1}}$ is a basis at each such $x^{\prime}$.

Example 3.4 Let $\mathcal{J}$ be the ideal in $\mathbb{C}^{4}$ generated by $\left(w_{1}^{2}, w_{2}^{2}, w_{1} w_{2}, w_{1} z_{2}-w_{2} z_{1}\right)$. It is readily checked that $\mathscr{O}_{X}$ is a free $\mathscr{O}_{Z}$-module at a point on $Z=\left\{w_{1}=w_{2}=0\right\}$ where $z_{1}$ or $z_{2}$ is $\neq 0$. If, say, $z_{1} \neq 0$, then we can take $1, w_{1}$ as generators. At the point $z=(0,0)$, e.g., $1, w_{1}, w_{2}$ form a minimal set of generators, and then $\mathscr{O}_{X}$ is not a free $\mathscr{O}_{Z}$-module, since there is a non-trivial relation between $w_{1}$ and $w_{2}$.

We claim that $\mathscr{O}_{X}$ has pure dimension. That is, we claim that there is no embedded associated prime ideal at $(0,0)$; since $Z$ is irreducible, this is the same as saying that $\mathcal{J}$ is primary with respect to $Z$. To see the claim, let $\phi$ and $\psi$ be functions such that $\phi \psi$ is in $\mathcal{J}$ and $\psi$ is not in $\sqrt{\mathcal{J}}$. The latter assumption means, in view of the Nullstellensatz, that $\psi$ does not vanish identically on $Z$, i.e., $\psi=a(z)+\mathscr{O}(w)$, where $a$ does not vanish identically. Since in particular $\phi \psi$ must vanish on $Z$ it follows that $\phi=\mathscr{O}(w)$. It is now easy to see that $\phi$ is in $\mathcal{J}$. We conclude that $\mathcal{J}$ is primary.

The pure-dimensionality of $\mathscr{O}_{X}$ can also be rephrased in the following way: If $\phi$ is holomorphic and is 0 generically, then $\phi=0$. If we delete the generator $w_{1} w_{2}$ from the definition of $\mathcal{J}$ in the example, then $\phi=w_{1} w_{2}$ is 0 generically in $\mathscr{O}_{\Omega} / \mathcal{J}$ but is not identically zero. Thus $\mathcal{J}$ then has an embedded primary ideal at $(0,0)$.

Example 3.5 Let $\Omega=\mathbb{C}_{z, w}^{2}$ and $\mathcal{J}=\left(w^{2}\right)$ so that $Z=\{w=0\}$. Then $1, w$ is a basis for $\mathscr{O}_{X}=\mathscr{O}_{\mathbb{C}^{2}} /\left(w^{2}\right)$ so each function $\phi$ in $\mathscr{O}_{X}$ has a unique representation $a_{0}(z) \otimes 1+a_{1}(z) \otimes w$. Let us consider the new coordinates $\zeta=z-w, \eta=w$. Then $\mathcal{J}=\left(\eta^{2}\right)$ and since

$a_{0}(z)+a_{1}(z) w=a_{0}(\zeta+\eta)+a_{1}(\zeta+\eta) \eta=a_{0}(\zeta)+\left(\partial a_{0} / \partial \zeta\right)(\zeta) \eta+a_{1}(\zeta) \eta+\mathcal{J}$

we have the representation $a_{0}(\zeta) \otimes 1+\left(a_{1}(\zeta)+\partial a_{0} / \partial \zeta\right)(\zeta) \otimes \eta$ with respect to $(\zeta, \eta)$

More generally, assume that, at a given point in $X_{\text {reg }} \subset \Omega$, we have two different choices $(z, w)$ and $(\zeta, \eta)$ of coordinates so that $Z=\{w=0\}=\{\eta=0\}$, and bases $1, \ldots, w^{\alpha_{\nu-1}}$ and $1, \ldots, \eta^{\beta_{v-1}}$ for $\mathscr{O}_{X}$ as a free module over $\mathscr{O}_{Z}$. Then there is a $v \times v$ matrix $L$ of holomorphic differential operators so that if $\left(a_{j}\right)$ is any tuple in $\left(\mathscr{O}_{Z}\right)^{v}$ and $\left(b_{j}\right)=L\left(a_{j}\right)$, then $a_{0} \otimes 1+\cdots+a_{\nu-1} \otimes w^{\alpha_{\nu-1}}=b_{0} \otimes 1+\cdots+b_{v-1} \otimes \eta^{\beta_{\nu-1}}+\mathcal{J}$. 


\section{Smooth $(0, *)$-forms on a non-reduced space $X$}

Let $i: X \rightarrow \Omega$ be a local embedding of $X$. In order to define the sheaf of smooth $(0, *)$-forms on $X$, in analogy with the reduced case, we have to state which smooth $(0, *)$-forms $\Phi$ in $\Omega$ "vanish" on $X$, or more formally, give a meaning to $i^{*} \Phi=0$. We will see, cf., Lemma 4.8 below, that the suitable requirement is that locally on $X_{\text {reg }}, \Phi$ belongs to $\mathscr{E}_{\Omega}^{0, *} \mathcal{J}+\mathscr{E}_{\Omega}^{0, *} \overline{\mathcal{J}}_{Z}+\mathscr{E}_{\Omega}^{0, *} d \overline{\mathcal{J}}_{Z}$, where $\mathcal{J}_{z}$ is the ideal sheaf defining $Z$. However, it turns out to be more convenient to represent the sheaf $\mathcal{K} e r i^{*}$ of such forms as the annihilator of certain residue currents, and this is the path we will follow. Moreover, these currents play a central role themselves later on.

The following classical duality result is fundamental for this paper; see, e.g., [3] for a discussion.

Proposition 4.1 If $\mathcal{J}$ has pure dimension, then

$$
\mathcal{J}=\operatorname{ann} \mathscr{O}_{\Omega} \mathcal{H} \operatorname{om}\left(\mathscr{O}_{\Omega} / \mathcal{J}, \mathcal{C} \mathcal{H}_{\Omega}^{Z}\right)
$$

That is, $\phi$ is in $\mathcal{J}$ if and only if $\phi \mu=0$ for all $\mu$ in $\mathcal{H o m}\left(\mathscr{O}_{\Omega} / \mathcal{J}, \mathcal{C} \mathcal{H}_{\Omega}^{Z}\right)$. It is also well-known, see, e.g., [3, Theorem 1.5], that

$$
\mathcal{H o m}\left(\mathscr{O}_{\Omega} / \mathcal{J}, \mathcal{C H}_{\Omega}^{Z}\right) \simeq \mathcal{E} x t^{p}\left(\mathscr{O}_{\Omega} / \mathcal{J}, K_{\Omega}\right)
$$

so $\mathcal{H}$ om $\left(\mathscr{O}_{\Omega} / \mathcal{J}, \mathcal{C H}_{\Omega}^{Z}\right)$ is a coherent analytic sheaf. Locally we thus have a finite number of generators $\mu^{1}, \ldots, \mu^{m}$. In Example 6.9, we compute explicitly such generators for the ideal $\mathcal{J}$ in Example 3.4.

Let $\xi$ be a smooth $(0, *)$-form in $\Omega$. Without first giving meaning to $i^{*}$, we define the sheaf $\mathcal{K}$ er $i^{*}$ by saying that $\xi$ is in $\mathcal{K}$ er $i^{*}$ if

$$
\xi \wedge \mu=0, \quad \mu \in \mathcal{H o m}\left(\mathscr{O}_{\Omega} / \mathcal{J}, \mathcal{C} \mathcal{H}_{\Omega}^{Z}\right)
$$

Notice that if $\xi$ is holomorphic, then, in view of the duality (4.1), $\xi$ is in $\mathcal{K} e r i^{*}$ if and only if $\xi$ is in $\mathcal{J}$.

Definition 4.2 We define the sheaf of smooth $(0, *)$-forms on $X$ as

$$
\mathscr{E}_{X}^{0, *}:=\mathscr{E}_{\Omega}^{0, *} / \mathcal{K} \text { er } i^{*}
$$

We will prove below that this sheaf is independent of the choice of embedding and thus intrinsic on $X$.

Given $\phi$ in $\mathscr{E}_{\Omega}^{0, *}$, let $i^{*} \phi$ be its image in $\mathscr{E}_{X}^{0, *}$. In particular, $i^{*} \xi=0$ means that $\xi$ belongs to $\mathcal{K} e r i^{*}$, which then motivates this notation. Notice that $\mathcal{K}$ er $i^{*}$ is a twosided ideal in $\mathscr{E}_{\Omega}^{0, *}$, i.e., if $\phi$ is in $\mathscr{E}_{\Omega}^{0, *}$ and $\xi$ is in $\mathcal{K} e r i^{*}$, then $\phi \wedge \xi$ and $\xi \wedge \phi$ are in $\mathcal{K} e r i^{*}$. It follows that we have an induced wedge product on $\mathscr{E}_{X}^{0, *}$ such that

$$
i^{*}(\phi \wedge \xi)=i^{*} \phi \wedge i^{*} \xi
$$


Remark 4.3 It follows from Lemma 4.8 below that in case $X=Z$ is reduced, then $\xi$ is in $\mathcal{K}$ er $i^{*}$ if and only its pullback to $X_{\text {reg }}$ vanishes. Thus our definition of $\mathscr{E}_{X}^{0, *}$ is consistent with the usual one in that case.

Lemma 4.4 Using the notation of (3.1),

$$
\iota_{*}: \mathcal{H o m}_{\mathscr{O}_{\widehat{\Omega}}}\left(\mathscr{O}_{\widehat{\Omega}} / \widehat{\mathcal{J}}, \mathcal{W}_{\widehat{\Omega}}^{Z}\right) \rightarrow \mathcal{H o m}_{\mathscr{O}_{\Omega}}\left(\mathscr{O}_{\Omega} / \mathcal{J}, \mathcal{W}_{\Omega}^{Z}\right)
$$

is an isomorphism.

We can realize the mapping in (4.4) as the tensor product $\tau \mapsto \tau \wedge[w=0]$, where $[w=0]$ is the Lelong current in $\Omega$ associated with the submanifold $\{w=0\}$.

Proof To begin with, $\iota_{*}$ maps pseudomeromorphic $(\hat{N}, \hat{p}+\ell)$-currents with support on $Z \subset \widehat{\Omega}$ to pseudomeromorphic $(N, p+\ell)$-currents with support on $Z \subset \Omega$. If, in addition, $\tau$ has the SEP with respect to $Z$, then $\iota_{*} \tau$ has, as well by (2.15). Moreover, if $\tau$ is annihilated by $\widehat{\mathcal{J}}$, then $\iota_{*} \tau$ is annihilated by $\mathcal{J}=\widehat{\mathcal{J}} \otimes 1+(w)$. Thus the mapping (4.4) is well-defined, and it is injective since $\iota$ is injective.

Now assume that $\mu$ is in $\mathcal{H} \operatorname{om}\left(\mathscr{O}_{\Omega} / \mathcal{J}, \mathcal{W}_{\Omega}^{Z}\right)$. Arguing as in the proof of Corollary 2.7, we see that $\mu=\iota_{*} \hat{\mu}$ for a current $\hat{\mu}$ in $\mathcal{W}_{\widehat{\Omega}}^{Z}$. Since $\widehat{\mathcal{J}}=\iota^{*} \mathcal{J}$ and $\mathcal{J} \mu=0$, it follows that $\widehat{\mathcal{J}} \hat{\mu}=0$. Thus (4.4) is surjective.

Since $\iota_{*}$ is injective, $\bar{\partial} \tau=0$ if and only if $\bar{\partial} \iota_{*} \tau=0$, and thus we get

Corollary 4.5 Using the notation of (3.1),

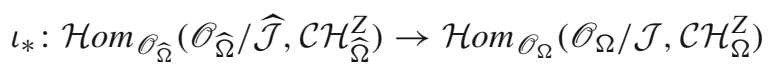

is an isomorphism.

Corollary 4.6 Using the notation in (3.1),

$$
\iota^{*}: \mathscr{E}_{\Omega}^{0, *} / \mathcal{K} e r i^{*} \rightarrow \mathscr{E}_{\widehat{\Omega}}^{0, *} / \mathcal{K} e r j^{*}
$$

is an isomorphism.

Proof It follows immediately from (4.5) that the mapping (4.6) is well-defined and injective. Given $\widehat{\xi}$ in $\mathscr{E} \widehat{\Omega}, *$, let $\xi=\widehat{\xi} \otimes 1$. Then $\iota^{*} \xi=\widehat{\xi}$ and so (4.6) is indeed surjective as well.

It follows from (4.6) and (4.3) that the sheaf $\mathscr{E}_{X}^{0, *}$ is intrinsically defined on $X$. Since $\bar{\partial}$ maps $\mathcal{K}$ er $i^{*}$ to $\mathcal{K}$ er $i^{*}$, we have a well-defined operator $\bar{\partial}: \mathscr{E}_{X}^{0, *} \rightarrow \mathscr{E}_{X}^{0, *+1}$ such that $\bar{\partial}^{2}=0$. Unfortunately the sheaf complex so obtained is not exact in general, see, e.g., [6, Example 1.1] for a counterexample already in the reduced case. 


\subsection{Local representation on $X_{\text {reg }}$ of smooth forms}

Recall that $X_{\text {reg }}$ is the open subset of $X$, where the underlying reduced space is smooth and $\mathscr{O}_{X}$ is Cohen-Macaulay. Let us fix some point in $X_{\text {reg }}$, and assume that we have local coordinates $(z, w)$ such that $Z=\{w=0\}$. We also choose generators $1, w^{\alpha_{1}}, \ldots, w^{\alpha_{v-1}}$ of $\mathscr{O}_{X}$ as a free $\mathscr{O}_{Z}$-module, which exist by Corollary 3.3, and generators $\mu^{1}, \ldots, \mu^{m}$ of $\mathcal{H} \operatorname{om}\left(\mathscr{O}_{\Omega} / \mathcal{J}, \mathcal{C H}_{\Omega}^{Z}\right)$.

Notice that for each smooth $(0, *)$-form $\Phi$ in $\Omega, \Phi \mapsto \Phi \wedge \mu^{\ell}$ only depends on its class $\phi$ in $\mathscr{E}_{X}^{0, *}$, and $\phi$ is in fact determined by these currents. By Proposition 2.5 each of these currents can (locally) be represented by a tuple of currents in $\mathcal{W}_{Z}^{0, *}$. Putting all these tuples together, we get a tuple in $\left(\mathcal{W}_{Z}^{0, *}\right)^{M}$, where $M=M_{1}+\cdots+M_{m}$ and $M_{j}$ is the number of indices in (2.11) in the representation of $\mu^{j}$.

Recall from Corollary 3.3 that $\phi$ in $\mathscr{O}_{X}$ has a unique representative

$$
\hat{\phi}=\hat{\phi}_{0}+\hat{\phi}_{1} \otimes w^{\alpha_{1}}+\cdots+\hat{\phi}_{\nu-1} \otimes w^{\alpha_{\nu-1}},
$$

where $\hat{\phi}_{j}$ are in $\mathscr{O}_{Z}$. We thus have an $\mathscr{O}_{Z}$-linear morphism

$$
T:\left(\mathscr{O}_{Z}\right)^{v} \rightarrow\left(\mathscr{O}_{Z}\right)^{M}
$$

The morphism is injective by Proposition 4.1, and the holomorphic matrix $T$ is therefore generically pointwise injective.

Lemma 4.7 Each $\phi$ in $\mathscr{E}_{X}^{0, *}$ has a unique representation $(4.7)$ where $\hat{\phi}_{j}$ are in $\mathscr{E}_{Z}^{0, *}$.

Proof To begin with notice that a given smooth $\phi$ must have at least one such representation. In fact, taking the finite Taylor expansion (2.13) we can forget about high order terms, since they must annihilate all the $\mu^{j}$, and the terms $\bar{w}$ and $d \bar{w}$ annihilate all the $\mu^{j}$ as well since they are pseudomeromorphic with support on $\{w=0\}$. On the other hand, each $w^{\alpha}$ not in the set of generators must be of the form

$$
w^{\alpha}=a_{0}+a_{1} \otimes w^{\alpha_{1}}+\cdots+a_{\nu-1} \otimes w^{\alpha_{\nu-1}}+\mathcal{J},
$$

and hence $\phi_{\alpha} \otimes w^{\alpha}$ is of the form (4.7). Thus the representation exists. To show uniqueness of the representation, we assume that $\hat{\phi}$ is in $\mathcal{K} e r i^{*}$. Then the tuple $\left(\hat{\phi}_{j}\right)$ is mapped to 0 by the matrix $T$, and since $T$ is generically pointwise injective we conclude that each $\hat{\phi}_{j}$ vanishes.

By the above proof we get

Lemma 4.8 A smooth $(0, *)$-form $\xi$ in $\Omega$ is in $\mathcal{K}$ er $i^{*}$ if and only if $\xi$ is in $\mathscr{E}_{\Omega}^{0, *} \mathcal{J}+$ $\mathscr{E}_{\Omega}^{0, *} \overline{\mathcal{J}}_{Z}+\mathscr{E}_{\Omega}^{0, *} d \overline{\mathcal{J}}_{Z}$ on $X_{\text {reg }}$, where $\mathcal{J}_{Z}$ is the radical sheaf of $Z$.

Remark 4.9 This is not the same as saying that $\xi$ is in $\mathscr{E}_{\Omega}^{0, *} \mathcal{J}+\mathscr{E}_{\Omega}^{0, *} \overline{\mathcal{J}}_{Z}+\mathscr{E}_{\Omega}^{0, *} d \overline{\mathcal{J}}_{Z}$ at singular points. For a simple counterexample, consider $\phi=x \bar{y}$ on the reduced space $Z=\{x y=0\} \subset \mathbb{C}^{2}$. 
However, this can happen also when $Z$ is irreducible at a point. For example, the variety $Z=\left\{x^{2} y-z^{2}=0\right\} \subset \mathbb{C}^{3}$ is irreducible at 0 , but there exist points arbitrarily close to 0 such that $(Z, z)$ is not irreducible. In this case, the ideal of smooth functions vanishing on $(Z, 0)$ is strictly larger than $\mathscr{E}_{\Omega}^{0,0} \mathcal{J}_{Z, 0}+\mathscr{E}_{\Omega}^{0,0} \overline{\mathcal{J}}_{Z, 0}$ see [26, Proposition 9, Chapter IV], and [25, Theorem 3.10, Chapter VI].

Remark 4.10 It is easy to check that if we have the setting as in the discussion at the end of Sect. 3 but $\left(a_{j}\right)$ is instead a tuple in $\mathscr{E}_{Z}^{0, *}$, then we can still define $\left(b_{j}\right)=L\left(a_{j}\right)$ if we consider the derivatives in $L$ as Lie derivatives; in fact, since $a_{j}$ has no holomorphic differentials, $L$ only acts on the smooth coefficients, and it is easy to check that $a_{0} \otimes 1+\cdots+a_{v-1} \otimes w^{\alpha_{\nu-1}}$ and $b_{0} \otimes 1+\cdots+b_{\nu-1} \otimes \eta^{\beta_{v-1}}$ are equal modulo $\mathscr{E}_{\Omega}^{0, *} \mathcal{J}+\mathscr{E}_{\Omega}^{0, *} \overline{\mathcal{J}}_{Z}+\mathscr{E}_{\Omega}^{0, *} d \overline{\mathcal{J}}_{Z}$, and thus define the same element in $\mathscr{E}_{X}^{0, *}$.

For future needs we prove in Sect. 6.1:

Lemma 4.11 The morphism $T$ is pointwise injective.

We can thus choose a holomorphic matrix $A$ such that

$$
0 \rightarrow \mathscr{O}_{Z}^{v} \stackrel{T}{\rightarrow} \mathscr{O}_{Z}^{M} \stackrel{A}{\rightarrow} \mathscr{O}_{Z}^{M^{\prime}}
$$

is pointwise exact, and we can also find holomorphic matrices $S$ and $B$ such that

$$
I=T S+B A .
$$

\section{Intrinsic $(n, *)$-currents on $X$}

In analogy with the reduced case we have the following definition when $X$ is possibly non-reduced.

Definition 5.1 The sheaf $\mathcal{C}_{X}^{n, q}$ of $(n, q)$-currents on $X$ is the dual sheaf of $(0, n-q)$ test forms, i.e., forms in $\mathscr{E}_{X}^{0, n-q}$ with compact support.

Here, just as in the case of reduced spaces, cf., for example [19, Section 4.2], the space of smooth forms $\mathscr{E}_{X}^{0, n-q}$ is equipped with the quotient topology induced by a local embedding.

More concretely, this means that given an embedding $i: X \rightarrow \Omega$, currents $\psi$ in $\mathcal{C}_{X}^{n, q}$ precisely correspond to the $(N, N-n+q)$-currents $\tau$ on $\Omega$ that vanish on $\mathcal{K} e r i^{*}$. Since $\mathcal{K}$ er $i^{*}$ is a two-sided ideal in $\mathscr{E}_{\Omega} 0, *$ this holds if and only if $\xi \wedge \tau=0$ for all $\xi$ in $\operatorname{Ker} i^{*}$. It is natural to write $\tau=i_{*} \psi$ so that

$$
i_{*} \psi \cdot \xi=\psi \cdot i^{*} \xi
$$

Clearly, we get a mapping $\bar{\partial}: \mathcal{C}_{X}^{n, q} \rightarrow \mathcal{C}_{X}^{n, q+1}$ such that $\bar{\partial}^{2}=0$.

Proposition 5.2 If $\tau$ is in $\mathcal{W}_{\Omega}^{Z}$ and $\mathcal{J} \tau=0$, then $\xi \wedge \tau=0$ for all smooth $\xi$ such that $i^{*} \xi=0$. 
Proof Because of the SEP it is enough to prove that $\xi \wedge \tau=0$ on $X_{\text {reg }}$. By assumption, $\mathcal{J}$ annihilates $\tau$, and by general properties of pseudomeromorphic currents, since $\tau$ has support on $Z, \overline{\mathcal{J}}_{Z}$ and $d \overline{\mathcal{J}}_{Z}$ annihilate $\tau$. Thus the proposition follows by Lemma 4.8.

Definition 5.3 An $(n, *)$-current $\psi$ on $X$ is in $\mathcal{W}_{X}^{n, *}$ if $i_{*} \psi$ is in $\mathcal{H o m}\left(\mathscr{O}_{\Omega} / \mathcal{J}, \mathcal{W}_{\Omega}^{Z}\right)$.

By definition we thus have the isomorphism

$$
i_{*}: \mathcal{W}_{X}^{n, *} \simeq \mathcal{H} \operatorname{om}\left(\mathscr{O}_{\Omega} / \mathcal{J}, \mathcal{W}_{\Omega}^{Z}\right)
$$

It follows from Lemma 4.4 that $\mathcal{W}_{X}^{n \text {,* }}$ is intrinsically defined.

Remark 5.4 By Corollary 2.7, this definition is consistent with the previous definition of $\mathcal{W}_{X}^{n, *}$ when $X$ is reduced. We cannot define $\mathcal{P} \mathcal{M}_{X}^{n, *}$ in the analogous simple way, cf., Remark 2.8 .

Definition 5.5 If $\psi$ is in $\mathcal{W}_{X}^{n, *}$ and $a$ is an almost semi-meromorphic (0,*)-current on $\Omega$ that is generically smooth on $Z$, then the product $a \wedge \psi$ is a current in $\mathcal{W}_{X}^{n \text {,* }}$ defined as follows: By definition, $i_{*} \psi$ is in $\mathcal{H o m}\left(\mathscr{O}_{\Omega} / \mathcal{J}, \mathcal{W}_{\Omega}^{Z}\right)$ and by Proposition 2.4 and (2.8), one can define $a \wedge i_{*} \psi$ in $\mathcal{H o m}\left(\mathscr{O}_{\Omega} / \mathcal{J}, \mathcal{W}_{\Omega}^{Z}\right)$; now $a \wedge \psi$ is the unique current in $\mathcal{W}_{X}^{n, *}$ such that $i_{*}(a \wedge \psi)=a \wedge i_{*} \psi$.

By (2.7),

$$
a \wedge \psi=\lim _{\epsilon \rightarrow 0^{+}} \chi\left(|h|^{2} / \epsilon\right) a \wedge \psi
$$

if $h$ cuts out the Zariski singular support of $a$.

Definition 5.6 We let $\omega_{X}^{n}$ be the sheaf of $\bar{\partial}$-closed currents in $\mathcal{W}_{X}^{n, 0}$.

This sheaf corresponds via $i_{*}$ to $\bar{\partial}$-closed currents in $\mathcal{H o m}\left(\mathscr{O}_{\Omega} / \mathcal{J}, \mathcal{W}_{\Omega}^{Z}\right)$ so we have the isomorphism

$$
i_{*}: \omega_{X}^{n} \simeq \mathcal{H} \operatorname{om}\left(\mathscr{O}_{\Omega} / \mathcal{J}, \mathcal{C} \mathcal{H}_{\Omega}^{Z}\right)
$$

When $X$ is reduced $\omega_{X}^{n}$ is the sheaf of $(n, 0)$-forms that are $\bar{\partial}$-closed in the BarletHenkin-Passare sense. Let $\mu^{1}, \ldots, \mu^{m}$ be a set of generators for $\mathcal{H}$ om $\left(\mathscr{O}_{\Omega} / \mathcal{J}, \mathcal{C} \mathcal{H}_{\Omega}^{Z}\right)$. They correspond via (5.3) to a set of generators $h^{1}, \ldots, h^{m}$ for the $\mathscr{O}_{X}$-module $\omega_{X}^{n}$.

We will also need a definition of $\mathcal{P} \mathcal{M}_{X}^{n, *}$. Let $\mathcal{F}_{X}$ be the subsheaf of $\mathcal{C}_{X}^{n, *}$ of $\tau$ such that $i_{*} \tau$ is in $\mathcal{P} \mathcal{M}_{\Omega}^{Z}$. If $\tau$ is a section of $\mathcal{F}_{X}$ and $W$ is a subvariety of some open subset of $Z$, then $\mathbf{1}_{W} i_{*} \tau$ is in $\mathcal{P} \mathcal{M}_{\Omega}^{Z}$, and by (2.3), $\mathbf{1}_{W} i_{*} \tau$ is annihilated by $\mathcal{K} e r i^{*}$. Hence we can define $\mathbf{1}_{W} \tau$ as the unique current in $\mathcal{F}_{X}$ such that $i_{*} \mathbf{1}_{W} \tau=\mathbf{1}_{W} i_{*} \tau$. Clearly, $\mathbf{1}_{W} \tau$ has support on $W$ and it is easily checked that the computational rule (2.3) holds also in $\mathcal{F}_{X}$. Moreover, $\mathcal{F}_{X}$ is closed under $\bar{\partial}$ since $\mathcal{P} \mathcal{M}_{\Omega}^{Z}$ is.

Definition 5.7 The sheaf $\mathcal{P} \mathcal{M}_{X}^{n \text {,* }}$ is the smallest subsheaf of $\mathcal{F}_{X}$ that contains $\mathcal{W}_{X}^{n \text {,* }}$ and is closed under $\bar{\partial}$ and multiplication by $\mathbf{1}_{W}$ for all germs $W$ of subvarieties of $Z$.

In view of Proposition 2.2 this definition coincides with the usual definition in case $X$ is reduced. It is readily checked that the dimension principle holds for $\mathcal{F}_{X}$, and hence it also holds for the (possibly smaller) sheaf $\mathcal{P} \mathcal{M}_{X}^{n, *}$, and in addition, (2.3) holds for forms $\xi$ in $\mathscr{E}_{X}^{0, *}$ and $\tau$ in $\mathcal{P} \mathcal{M}_{X}^{n, *}$. 


\section{Structure form on $X$}

Let $i: X \rightarrow \Omega \subset \mathbb{C}^{N}$ be a local embedding as before, let $p=N-n$ be the codimension of $X$, and let $\mathcal{J}$ be the associated ideal sheaf on $\Omega$. In a slightly smaller set, still denoted $\Omega$, there is a free resolution

$$
0 \rightarrow \mathscr{O}\left(E_{N_{0}}\right) \stackrel{f_{N_{0}}}{\longrightarrow} \cdots \stackrel{f_{3}}{\longrightarrow} \mathscr{O}\left(E_{2}\right) \stackrel{f_{2}}{\longrightarrow} \mathscr{O}\left(E_{1}\right) \stackrel{f_{1}}{\longrightarrow} \mathscr{O}\left(E_{0}\right)
$$

of $\mathscr{O}_{\Omega} / \mathcal{J}$; here $E_{k}$ are trivial vector bundles over $\Omega$ and $E_{0}$ is the trivial line bundle. This resolution induces a complex of vector bundles

$$
0 \rightarrow E_{N_{0}} \stackrel{f_{N_{0}}}{\longrightarrow} \cdots \stackrel{f_{3}}{\longrightarrow} E_{2} \stackrel{f_{2}}{\longrightarrow} E_{1} \stackrel{f_{1}}{\longrightarrow} E_{0}
$$

that is pointwise exact outside $Z$. Let $X_{k}$ be the set where $f_{k}$ does not have optimal rank. Then

$$
\cdots \subset X_{k+1} \subset X_{k} \subset \cdots \subset X_{p+1} \subset X_{p}=\cdots=X_{1}=Z
$$

these sets are independent of the choice of resolution and thus invariants of $\mathscr{O}_{\Omega} / \mathcal{J}$. Since $\mathscr{O}_{\Omega} / \mathcal{J}$ has pure codimension $p$,

$$
\operatorname{codim} X_{k} \geq k+1, \quad \text { for } k \geq p+1,
$$

see [14, Corollary 20.14]. Thus there is a free resolution (6.1) if and only if $X_{k}=\varnothing$ for $k>N_{0}$. Unless $n=0$ (which is not interesting in relation to the $\bar{\partial}$-equation), we can thus choose the resolution so that $N_{0} \leq N-1$. The variety $X$ is Cohen-Macaulay at a point $x$, i.e., the sheaf $\mathscr{O}_{\Omega} / \mathcal{J}$ is Cohen-Macaulay at $x$, if and only if $x \notin X_{p+1}$. Notice that $Z \backslash\left(X_{\text {reg }}\right)_{\text {red }}=Z_{\text {sing }} \cup X_{p+1}$. The sets $X_{k}$ are independent of the choice of embedding, see [9, Lemma 4.2], and are thus intrinsic subvarieties of $Z=X_{\text {red }}$, and they reflect the complexity of the singularities of $X$.

Let us now choose Hermitian metrics on the bundles $E_{k}$. We then refer to (6.1) as a Hermitian resolution of $\mathscr{O}_{\Omega} / \mathcal{J}$ in $\Omega$. In $\Omega \backslash X_{k}$ we have a well-defined vector bundle morphism $\sigma_{k+1}: E_{k} \rightarrow E_{k+1}$, if we require that $\sigma_{k+1}$ vanishes on $\left(\operatorname{Im} f_{k+1}\right)^{\perp}$, takes values in $\left(\mathcal{K} e r f_{k+1}\right)^{\perp}$, and that $f_{k+1} \sigma_{k+1}$ is the identity on $\operatorname{Im} f_{k+1}$. Following [7, Section 2] we define smooth $E_{k}$-valued forms

$$
u_{k}=\left(\bar{\partial} \sigma_{k}\right) \cdots\left(\bar{\partial} \sigma_{2}\right) \sigma_{1}=\sigma_{k}\left(\bar{\partial} \sigma_{k-1}\right) \cdots\left(\bar{\partial} \sigma_{1}\right)
$$

in $\Omega \backslash X$; for the second equality, see [7, (2.3)]. We have that

$$
f_{1} u_{1}=1, \quad f_{k+1} u_{k+1}-\bar{\partial} u_{k}=0, \quad k \geq 1,
$$

in $\Omega \backslash X$. If $f:=\oplus f_{k}$ and $u:=\sum u_{k}$, then these relations can be written economically as $\nabla_{f} u=1$, where $\nabla_{f}:=f-\bar{\partial}$. To make the algebraic machinery work properly one has to introduce a superstructure on the bundle $E=: \oplus E_{k}$ so that vectors in $E_{2 k}$ are 
even and vectors in $E_{2 k+1}$ are odd; hence $f, \sigma:=\oplus \sigma_{k}$, and $u:=\sum u_{k}$ are odd. For details, see [7]. It turns out that $u$ has a (necessarily unique) almost semi-meromorphic extension $U$ to $\Omega$. The residue current $R$ is defined by the relation

$$
\nabla_{f} U=1-R
$$

It follows directly that $R$ is $\nabla_{f}$-closed. In addition, $R$ has support on $Z$ and is a sum $\sum R_{k}$, where $R_{k}$ is a pseudomeromorphic $E_{k}$-valued current of bidegree $(0, k)$. It follows from the dimension principle that $R=R_{p}+R_{p+1}+\cdots+R_{N}$. If we choose a free resolution that ends at level $N-1$, then $R_{N}=0$. If $X$ is Cohen-Macaulay and $N_{0}=p$ in (6.1), then $R=R_{p}$, and the $\nabla_{f}$-closedness implies that $R$ is $\bar{\partial}$-closed.

If $\phi$ is in $\mathcal{J}$ then $\phi R=0$ and in fact, $\mathcal{J}=$ ann $R$, see [7, Theorem 1.1].

Remark 6.1 In case $\mathcal{J}$ is generated by the single non-trivial function $f$, then we have the free resolution $0 \rightarrow \mathscr{O}_{\Omega} \stackrel{f}{\rightarrow} \mathscr{O}_{\Omega} \rightarrow \mathscr{O}_{\Omega} /(f) \rightarrow 0$; thus $U$ is just the principal value current $1 / f$ and $R=\bar{\partial}(1 / f)$. More generally, if $f=\left(f_{1}, \ldots, f_{p}\right)$ is a complete intersection, then

$$
R=\bar{\partial} \frac{1}{f_{p}} \wedge \cdots \wedge \bar{\partial} \frac{1}{f_{1}},
$$

where the right hand side is the so-called Coleff-Herrera product of $f$, see for example [1, Corollary 3.5].

There are almost semi-meromorphic $\alpha_{k}$ in $\Omega$, cf., [7, Section 2] and the proof of [6, Proposition 3.3], that are smooth outside $X_{k}$, such that

$$
R_{k+1}=\alpha_{k+1} R_{k}
$$

outside $X_{k+1}$ for $k \geq p$. In view of (6.3) and the dimension principle, $\mathbf{1}_{X_{k+1}} R_{k+1}=0$ and hence (6.6) holds across $X_{k+1}$, i.e., $R_{k+1}$ is indeed equal to the product $\alpha_{k+1} R_{k}$ in the sense of Proposition 2.1. In particular, it follows that $R_{k}$ has the SEP with respect to $Z$.

In this section, we let $\left(z_{1}, \ldots, z_{N}\right)$ denote coordinates on $\mathbb{C}^{N}$, and let $d z:=d z_{1} \wedge$ $\cdots \wedge d z_{N}$.

Lemma 6.2 There is a matrix of almost semi-meromorphic currents $b$ such that

$$
R \wedge d z=b \mu
$$

where $\mu$ is a tuple of currents in $\mathcal{H o m}\left(\mathscr{O}_{\Omega} / \mathcal{J}, \mathcal{C H}_{\Omega}^{Z}\right)$.

Proof As in [6, Section 3], see also [32, Proposition 3.2], one can prove that $R_{p}=$ $\sigma_{F} \mu$, where $\mu$ is a tuple of currents in $\mathcal{H o m}\left(\mathscr{O}_{\Omega} / \mathcal{J}, \mathcal{C H} Z\right.$ and $\sigma_{F}$ is an almost semimeromorphic current that is smooth outside $X_{p+1}$.

Let $b_{p}=\sigma_{F}$ and $b_{k}=\alpha_{k} \cdots \alpha_{p+1} \sigma_{F}$ for $k \geq p+1$. Then each $b_{k}$ is almost semi-meromorphic, cf., [10, Section 4.1]. In view of (6.6) we have that $R_{k}=b_{k} \mu$ outside $X_{p+1}$ since $b_{k}$ is smooth there. It follows by the SEP that it holds across $X_{p+1}$ as well since $R_{k}$ has the SEP with respect to $Z$. We then take $b=b_{p}+b_{p+1}+\cdots$. 
By Proposition 2.4 we get

Corollary 6.3 The current $R \wedge d z$ is in $\mathcal{H o m}\left(\mathscr{O}_{\Omega} / \mathcal{J}, \mathcal{W}_{\Omega}^{Z}\right)$.

From Lemma 6.2, Corollary 6.3, (5.1), and (5.3) we get the following analogue to [6, Proposition 3.3]:

Proposition 6.4 Let (6.1) be a Hermitian resolution of $\mathscr{O}_{\Omega} / \mathcal{J}$ in $\Omega$, and let $R$ be the associated residue current. Then there exists a (unique) current $\omega$ in $\mathcal{W}_{X}^{n, *}$ such that

$$
i_{*} \omega=R \wedge d z
$$

There is a matrix $b$ of almost semi-meromorphic $(0, *)$-currents in $\Omega$, smooth outside of $X_{p+1}$, and a tuple $\vartheta$ of currents in $\omega_{X}^{n}$ such that

$$
\omega=b \vartheta
$$

More precisely, $\omega=\omega_{0}+\omega_{1}+\cdots+\omega_{n},{ }^{1}$ where $\omega_{k} \in \mathcal{W}^{n, k}\left(X, E_{p+k}\right)$, and if $f^{j}:=f_{p+j}$, then

$$
f^{0} \omega_{0}=0, \quad f^{j+1} \omega_{j+1}-\bar{\partial} \omega_{j}=0, \text { for } j \geq 0 .
$$

We will also use the short-hand notation $\nabla_{f} \omega=0$. As in the reduced case, following [6], we say that $\omega$ is a structure form for $X$. The products in (6.9) are defined according to Definition 5.5.

Remark 6.5 Recall that $X_{p+1}=\emptyset$ if $X$ is Cohen-Macaulay, so in that case $\omega=b \vartheta$, where $b$ is smooth. If we take a free resolution of length $p$, then $\omega=\omega_{0}$, and $\bar{\partial} \omega_{0}=$ $f^{1} \omega_{1}=0$, so $\omega$ is in $\omega_{X}^{n}$.

Remark 6.6 If $X=\{f=0\}$ is a reduced hypersurface in $\Omega$, then $R=\bar{\partial}(1 / f)$ and $\omega$ is the classical Poincaré residue form on $X$ associated with $f$, which is a meromorphic form on $X$. More generally, if $X$ is reduced, since forms in $\omega_{X}^{n}$ are then meromorphic, by (6.9), $\omega$ can be represented by almost semi-meromorphic forms on $X$.

We now consider the case when $X$ is non-reduced. We recall that a differential operator is a Noetherian operator for an ideal $\mathcal{J}$ if $\mathcal{L} \varphi \in \sqrt{\mathcal{J}}$ for all $\varphi \in \mathcal{J}$. It is proved by Björk, [13], see also [32, Theorem 2.2], that if $\mu \in \mathcal{H}$ om $\left(\mathscr{O}_{\Omega} / \mathcal{J}, \mathcal{C} \mathcal{H}_{\Omega}^{Z}\right)$, then there exists a Noetherian operator $\mathcal{L}$ for $\mathcal{J}$ with meromorphic coefficients such that the action of $\mu$ on $\xi$ equals the integral of $\mathcal{L} \xi$ over $Z$. By (5.3), the action of $h$ in $\omega_{X}^{n}$ on $\xi$ in $\mathscr{E}_{X}^{0, *}$ can then be expressed as

$$
h . \xi=\int_{Z} \mathcal{L} \xi .
$$

\footnotetext{
1 In [6, Proposition 3.3], the sum ends with $\omega_{n-1}$ instead of $\omega_{n}$, which, as remarked above, one can indeed assume when $n \geq 1$ and the resolution is chosen to be of length $\leq N-1$.
} 
One can then verify using this formula and (6.9) that the action of the structure form $\omega$ on a test form $\xi$ in $\mathscr{E}_{X}^{0, *}$ equals

$$
\omega . \xi=\int_{Z} \tilde{\mathcal{L}} \xi,
$$

where $\tilde{\mathcal{L}}$ is now a tuple of Noetherian operators for $\mathcal{J}$ with almost semi-meromorphic coefficients, cf., [32, Section 4].

Notice that (6.1) gives rise to the dual Hermitian complex

$$
0 \rightarrow \mathscr{O}\left(E_{0}^{*}\right) \stackrel{f_{1}^{*}}{\rightarrow} \cdots \rightarrow \mathscr{O}\left(E_{p-1}^{*}\right) \stackrel{f_{p}^{*}}{\rightarrow} \mathscr{O}\left(E_{p}^{*}\right) \stackrel{f_{p+1}^{*}}{\rightarrow} \cdots
$$

Let $\xi=\xi_{0} \wedge d z$ be a holomorphic section of the sheaf

$$
\mathcal{H o m}\left(E_{p}, K_{\Omega}\right) \simeq \mathscr{O}\left(E_{p}^{*}\right) \otimes \mathscr{O}\left(K_{\Omega}\right)
$$

such that $f_{p+1}^{*} \xi_{0}=0$. Then $\bar{\partial}\left(\xi_{0} \omega_{0}\right)= \pm \xi_{0} \bar{\partial} \omega_{0}= \pm \xi_{0} f_{p+1} \omega_{1}= \pm\left(f_{p+1}^{*} \xi_{0}\right) \omega_{1}=0$, so that $\xi_{0} \omega_{0}$ is in $\omega_{X}^{n}$. Moreover, if $\xi_{0}=f_{p}^{*} \eta$ for $\eta$ in $\mathscr{O}\left(E_{p-1}^{*}\right)$, then $\xi_{0} \omega_{0}=f_{p}^{*} \eta \omega_{0}=$ $\pm \eta f_{p} \omega_{0}=0$. We thus have a sheaf mapping

$$
\mathcal{H}^{p}\left(\mathcal{H} \operatorname{om}\left(E_{\bullet}, K_{\Omega}\right)\right) \rightarrow \omega_{X}^{n}, \quad \xi_{0} \wedge d z \mapsto \xi_{0} \omega_{0}
$$

Proposition 6.7 The mapping (6.12) is an isomorphism, which establishes an intrinsic isomorphism

$$
\mathcal{E}_{x}{ }^{p}\left(\mathscr{O}_{\Omega} / \mathcal{J}, K_{\Omega}\right) \simeq \omega_{X}^{n} .
$$

Proof If $h$ is in $\omega_{X}^{n}$, then $i_{*} h$ is in $\mathcal{H o m}\left(\mathscr{O}_{\Omega} / \mathcal{J}, \mathcal{C} \mathcal{H}_{\Omega}^{Z}\right)$. We have mappings

$$
\mathcal{H}^{p}\left(\mathcal{H o m}\left(E_{\bullet}, K_{\Omega}\right)\right) \rightarrow \omega_{X}^{n} \stackrel{\simeq}{\rightarrow} \mathcal{H o m}\left(\mathscr{O}_{\Omega} / \mathcal{J}, \mathcal{C H}_{\Omega}^{Z}\right)
$$

where the first mapping is (6.12), and the second is $h \mapsto i_{*} h$. In view of (6.8), the composed mapping is $\xi=\xi_{0} \wedge d z \mapsto \xi R_{p}=\xi_{0} R_{p} \wedge d z$. $^{2}$ This mapping is an intrinsic isomorphism

$$
\operatorname{Ext}^{p}\left(\mathscr{O}_{\Omega} / \mathcal{J}, K_{\Omega}\right) \simeq \mathcal{H} \operatorname{om}\left(\mathscr{O}_{\Omega} / \mathcal{J}, \mathcal{C H}_{\Omega}^{Z}\right)
$$

according to [3, Theorem 1.5]. It follows that (6.12) also establishes an intrinsic isomorphism.

In particular it follows that $\omega_{X}^{n}$ is coherent, and we have:

If $\xi^{1}, \ldots, \xi^{m}$ are generators of $\left.\mathcal{H}^{p}\left(\mathcal{H o m}\left(E_{\bullet}^{*}, K_{\Omega}\right)\right)\right)$, where $\xi^{\ell}=\xi_{0}^{\ell} \wedge d z$, then $h^{\ell}:=\xi_{0}^{\ell} \omega_{0}, \ell=1, \ldots, m$, generate the $\mathscr{O}_{X}$-module $\omega_{X}^{n}$, and $\mu^{\ell}=i_{*} h^{\ell}=\xi^{\ell} R_{p}$ generate the $\mathscr{O}_{\Omega}$-module $\mathcal{H o m}\left(\mathscr{O}_{\Omega} / \mathcal{J}, \mathcal{C H}_{\Omega}^{Z}\right)$.

\footnotetext{
2 There is a superstructure involved, with respect to which $R_{p}$ has even degree, and therefore $d z \wedge R_{p}=$ $R_{p} \wedge d z$, explaining the lack of a sign in the last equality, see [6] or [7].
} 
Remark 6.8 The isomorphism

$$
\mathcal{H}^{p}\left(\mathcal{H o m}\left(E_{\bullet}, K_{\Omega}\right)\right) \stackrel{\simeq}{\rightarrow} \mathcal{H o m}\left(\mathscr{O}_{\Omega} / \mathcal{J}, \mathcal{C H}_{\Omega}^{Z}\right)
$$

was well-known since long ago, the contribution in [3] was the realization $\xi \mapsto \xi R_{p}$. $\square$

We give here an example where we can explicitly compute generators of $\mathcal{H o m}\left(\mathscr{O}_{\Omega} / \mathcal{J}, \mathcal{C} \mathcal{H}_{\Omega}^{Z}\right)$

Example 6.9 Let $\mathcal{J}$ be as in Example 3.4. We claim that $\mathcal{H o m}\left(\mathscr{O}_{\Omega} / \mathcal{J}, \mathcal{C H}_{\Omega}^{Z}\right)$ is generated by

$\mu_{1}:=\bar{\partial} \frac{1}{w_{1}} \wedge \bar{\partial} \frac{1}{w_{2}} \wedge d z \wedge d w$ and $\mu_{2}:=\left(z_{1} \bar{\partial} \frac{1}{w_{1}^{2}} \wedge \bar{\partial} \frac{1}{w_{2}}+z_{2} \bar{\partial} \frac{1}{w_{1}} \wedge \bar{\partial} \frac{1}{w_{2}^{2}}\right) \wedge d z \wedge d w$

In order to prove this claim, we use the comparison formula for residue currents from [21], which states that if $\mathscr{O}\left(F_{\bullet}\right)$ and $\mathscr{O}\left(E_{\bullet}\right)$ are free resolutions of $\mathscr{O}_{\Omega} / \mathcal{I}$ and $\mathscr{O}_{\Omega} / \mathcal{J}$, respectively, where $\mathcal{I}$ and $\mathcal{J}$ have codimension $\geq p$, and $a: F_{\bullet} \rightarrow E_{\bullet}$ is a morphism of complexes, then there exists a $\mathcal{H o m}\left(F_{0}, E_{p+1}\right)$-valued current $M_{p+1}$ such that $R_{p}^{E} a_{0}=a_{p} R_{p}^{F}+f_{p+1} M_{p+1}$. If $\xi$ is in $\mathcal{K} e r f_{p+1}^{*}$, we thus get that

$$
\xi R_{p}^{E} a_{0}=\xi a_{p} R_{p}^{F}
$$

We will apply this with $\mathscr{O}_{\Omega}\left(E_{\bullet}\right)$ as the free resolution

$$
0 \rightarrow \mathscr{O}_{\Omega} \stackrel{f_{3}}{\longrightarrow} \mathscr{O}_{\Omega}^{4} \stackrel{f_{2}}{\longrightarrow} \mathscr{O}_{\Omega}^{4} \stackrel{f_{1}}{\longrightarrow} \mathscr{O}_{\Omega} \rightarrow \mathscr{O}_{\Omega} / \mathcal{J} \rightarrow 0
$$

where

$$
\begin{aligned}
& f_{3}=\left[\begin{array}{c}
w_{2} \\
-w_{1} \\
z_{2} \\
-z_{1}
\end{array}\right], f_{2}=\left[\begin{array}{cccc}
z_{2} & 0 & -w_{2} & 0 \\
-z_{1} & z_{2} & w_{1} & -w_{2} \\
0 & -z_{1} & 0 & w_{1} \\
-w_{1} & -w_{2} & 0 & 0
\end{array}\right] \quad \text { and } \\
& f_{1}=\left[\begin{array}{lll}
w_{1}^{2} w_{1} w_{2} & w_{2}^{2} & z_{2} w_{1}-z_{1} w_{2}
\end{array}\right],
\end{aligned}
$$

and the Koszul complex $\left(F, \delta_{\mathbf{w}^{2}}\right)$ generated by $\mathbf{w}^{2}:=\left(w_{1}^{2}, w_{2}^{2}\right)$, which is a free resolution of $\mathscr{O} /\left(w_{1}^{2}, w_{2}^{2}\right)$. We then take the morphism of complexes $a: F_{\bullet} \rightarrow E_{\bullet}$ given by 


$$
a_{2}=\left[\begin{array}{c}
0 \\
0 \\
w_{2} \\
w_{1}
\end{array}\right], \quad a_{1}=\left[\begin{array}{ll}
1 & 0 \\
0 & 0 \\
0 & 1 \\
0 & 0
\end{array}\right] \text { and } a_{0}=[1]
$$

Since the current $R_{2}^{F}$ is equal to the Coleff-Herrera product $\bar{\partial}\left(1 / w_{1}^{2}\right) \wedge \bar{\partial}\left(1 / w_{2}^{2}\right)$, cf., Remark 6.1, we thus get by (6.16) and Remark 6.8 that $\mathcal{H o m}\left(\mathscr{O}_{\Omega} / \mathcal{J}, \mathcal{C H}_{\Omega}^{Z}\right)$ is generated by

$$
\left(\mathcal{K} e r f_{3}^{*}\right) a_{2} \bar{\partial} \frac{1}{w_{1}^{2}} \wedge \bar{\partial} \frac{1}{w_{2}^{2}} .
$$

A straightforward calculation gives the generators $\mu_{1}$ and $\mu_{2}$ above.

\subsection{Proof of Lemma 4.11}

Since $T$ is generically injective, it is clearly injective if $n=0$. We are going to reduce to this case. Fix the point $0 \in Z$ and let $\mathcal{I}$ be the ideal generated by $z=\left(z_{1}, \ldots, z_{n}\right)$.

Let $\mathscr{O}\left(E_{\bullet}\right)$ be a free Hermitian resolution of $\mathscr{O}_{\Omega} / \mathcal{J}$ of minimal length $p=N-n$ at 0 and let $R^{E}$ be the associated residue current. Recall that the canonical isomorphism (6.15) is realized by $\xi \mapsto \xi R_{p}^{E}$. Let $F_{\bullet}$ be the Koszul complex generated by $z$; then $\mathscr{O}\left(F_{\bullet}\right)$ is a free resolution of $\mathscr{O}_{\Omega} / \mathcal{I}$. Since $\mathcal{J}$ and $\mathcal{I}$ are Cohen-Macaulay and intersect properly in $\Omega$, the complex $\mathscr{O}_{\Omega}((E \otimes F) \bullet)$ is a free resolution of $\mathscr{O}_{\Omega} /(\mathcal{J}+\mathcal{I})$, and the corresponding residue current is

$$
R_{N}^{E \otimes F}=R_{p}^{E} \wedge R_{n}^{F}
$$

according to [2, Theorem 4.2]. From [3, Theorem 1.5] again it follows that the canonical isomorphism

$$
\mathcal{H}^{N}\left(\mathcal{H o m}\left((E \otimes F)_{\bullet}, K_{\Omega}\right)\right) \rightarrow \mathcal{H} \operatorname{om}\left(\mathscr{O}_{\Omega} /(\mathcal{J}+\mathcal{I}), \mathcal{C H}_{\Omega}^{\{0\}}\right)
$$

is given by $\eta \mapsto \eta R_{N}^{E \otimes F}$.

Let $\mu^{1}, \ldots, \mu^{m}$ be a minimal set of generators for $\mathcal{H o m}\left(\mathscr{O}_{\Omega} / \mathcal{J}, \mathcal{C H}_{\Omega}^{Z}\right)$ at 0 . Then $\mu^{j}=\xi^{j} R_{p}^{E}$, where $\xi^{j}$ is a minimal set of generators for $\mathcal{H}^{p}\left(\mathcal{H} \operatorname{Hom}\left(E_{\bullet}, K_{\Omega}\right)\right)$. Notice that

$$
\mathcal{H}^{N}\left(\mathcal{H o m}\left((E \otimes F)_{\bullet}, K_{\Omega}\right)\right)=\mathcal{H}^{p}\left(\mathcal{H o m}\left(E_{\bullet}, K_{\Omega}\right)\right) \otimes_{\mathscr{O}} \mathcal{H}^{n}\left(\mathcal{H}_{o m}\left(F_{\bullet}, \mathscr{O}_{\Omega}\right)\right)
$$

Since $\mathcal{H}^{n}\left(\mathcal{H} \operatorname{om}\left(F_{\bullet}, \mathscr{O}_{\Omega}\right)\right)$ is generated by 1 , it follows that $\mathcal{H}^{N}\left(\mathcal{H} o m\left((E \otimes F) \bullet, K_{\Omega}\right)\right)$ is generated by $\xi^{j} \otimes 1$. We conclude that $\mathcal{H o m}\left(\mathscr{O}_{\Omega} /(\mathcal{J}+\mathcal{I}), \mathcal{C} \mathcal{H}_{\Omega}^{\{0\}}\right)$ is generated by $\xi^{j} \otimes 1 \cdot R_{p}^{E} \wedge R_{n}^{F}=\mu^{j} \wedge \mu^{z}, j=1, \ldots, m$, where $R_{n}^{F}=\mu^{z}=\check{\partial}\left(1 / z^{1}\right)$.

If $1, \ldots, w^{\alpha_{\nu-1}}$ is a basis for $\mathscr{O}_{\Omega} / \mathcal{J}$ as an $\mathscr{O}_{Z}$-module, then it is also a basis for $\mathscr{O}_{X_{0}}:=\mathscr{O}_{\Omega} /(\mathcal{J}+\mathcal{I})$ as a module over $\mathscr{O}_{\{0\}} \simeq \mathbb{C}$. Since $\phi \bar{\partial}\left(1 / z^{\mathbf{1}}\right)=\phi(0, \cdot) \bar{\partial}\left(1 / z^{\mathbf{1}}\right)$ 
we have that

$$
\begin{aligned}
\phi(z, w) \mu^{j} \wedge \mu^{z} & =\phi(z, w) \sum a_{\ell}^{j}(z) \bar{\partial} \frac{1}{w^{\ell+\mathbf{1}}} \wedge \bar{\partial} \frac{1}{z^{\mathbf{1}}} \\
& =\phi(0, w) \sum a_{\ell}^{j}(0) \bar{\partial} \frac{1}{w^{\ell+\mathbf{1}}} \wedge \bar{\partial} \frac{1}{z^{\mathbf{1}}}
\end{aligned}
$$

The morphism constructed in (4.8) for $X_{0}$ instead of $X$ is then $T_{0}=T(0)$, where $T$ is the morphism (4.8) for $X$. Thus $T(0)$ is injective.

\section{The intrinsic sheaf $\mathcal{W}_{X}^{0, *}$ on $X$}

Our aim is to find a fine resolution of $\mathscr{O}_{X}$ and since the complex (1.1) is not exact in general when $X$ is singular we have to consider larger fine sheaves; we first define sheaves $\mathcal{W}_{X}^{0, *} \supset \mathscr{E}_{X}^{0, *}$ of $(0, *)$-currents. Given a local embedding $i: X \rightarrow \Omega$ at a point on $X_{\text {reg }}$ and local coordinates $(z, w)$ as before, it is natural, in view of Lemma 4.7, to require that an element in $\mathcal{W}_{X}^{0, *}$ shall have a unique representation

$$
\phi=\widehat{\phi}_{0} \otimes 1+\widehat{\phi}_{1} \otimes w^{\alpha_{1}}+\cdots+\widehat{\phi}_{\nu-1} \otimes w^{\alpha_{\nu-1}}
$$

where $\widehat{\phi}_{j}$ are in $\mathcal{W}_{Z}^{0, *}$. In view of Remark 4.10 we should expect that the same transformation rules hold as for smooth $(0, *)$-forms. In particular it is then necessary that $\mathcal{W}_{Z}^{0 . *}$ is closed under the action of holomorphic differential operators, which in fact is true, see Proposition 7.11 below. We must also define a reasonable extension of these sheaves across $X_{\text {sing }}$. Before we present our formal definition we make a preliminary observation.

Lemma 7.1 If $\phi$ has the form (7.1) and $\tau$ is in $\mathcal{H o m}\left(\mathscr{O}_{\Omega} / \mathcal{J}, \mathcal{C H} \mathcal{H}_{\Omega}^{Z}\right)$, expressed in the form (2.11), then

$$
\phi \wedge \tau:=\sum_{i} \sum_{\gamma \geq \alpha_{i}} \widehat{\phi}_{i} \wedge \tau_{\gamma} \wedge d z \otimes \bar{\partial} \frac{d w}{w^{\gamma-\alpha_{i}+1}}
$$

is in $\mathcal{H o m}\left(\mathscr{O}_{\Omega} / \mathcal{J}, \mathcal{W}_{\Omega}^{Z}\right)$.

Proof The right hand side defines a current in $\mathcal{W}_{\Omega}^{Z}$ since $\widehat{\phi}_{i}$ are in $\mathcal{W}_{Z}^{0, *}$ and $\tau_{\gamma}$ are in $\mathscr{O}_{Z}$. We have to prove that it is annihilated by $\mathcal{J}$. Take $\xi$ in $\mathcal{J}$. On the subset of $Z$ where $\widehat{\phi}_{0}, \ldots, \widehat{\phi}_{\nu-1}$ are all smooth, $\phi \wedge \tau$, as defined above, is just multiplication of the smooth form $\phi$ by $\tau$, and thus $\xi \phi \wedge \tau=0$ there. We have a unique representation

$$
\xi \phi \wedge \tau=\sum_{\ell \geq 0} a_{\ell}(z) \wedge d z \otimes \bar{\partial} \frac{d w}{w^{\ell+1}},
$$

with $a_{\ell}$ in $\mathcal{W}_{Z}^{0, *}$. Since $a_{\ell}$ vanish on the set where all $\widehat{\phi}_{j}$ are smooth, we conclude from Proposition 2.9 that $a_{\ell}$ vanish identically. It follows that $\xi \phi \wedge \tau=0$. 
If $\phi$ has the form (7.1) in a neighborhood of some point $x \in X_{\text {reg }}$ and $h$ is in $\omega_{X}^{n}$, then we get an element $\phi \wedge h$ in $\mathcal{W}_{X}^{n, *}$ defined by $i_{*}(\phi \wedge h)=\phi \wedge i_{*} h$. It follows that $\phi$ in this way defines an element in $\mathcal{H o m}_{\mathscr{O}_{X}}\left(\omega_{X}^{n}, \mathcal{W}_{X}^{n, *}\right)$. This sheaf is global and invariantly defined and so we can make the following global definition.

Definition 7.2 $\mathcal{W}_{X}^{0, *}=\mathcal{H}_{\text {om }_{X}}\left(\omega_{X}^{n}, \mathcal{W}_{X}^{n, *}\right)$.

If $\phi$ is in $\mathcal{W}_{X}^{0, *}$ and $h$ is in $\omega_{X}^{n}$, we consider $\phi(h)$ as the product of $\phi$ and $h$, and sometimes write it as $\phi \wedge h$.

Since $\mathcal{W}_{X}^{n, *}$ are $\mathscr{E}_{X}^{0, *}$-modules, $\mathcal{W}_{X}^{0, *}$ are as well. Before we investigate these sheaves further, we give some motivation for the definition. First notice that we have a natural injection, cf., Proposition 4.1,

$$
\mathscr{O}_{X} \rightarrow \mathcal{H o m}\left(\omega_{X}^{n}, \omega_{X}^{n}\right), \quad \phi \mapsto(h \mapsto \phi h)
$$

Theorem 7.3 The mapping (7.3) is an isomorphism in the Zariski-open subset of $X$ where it is $S_{2}$.

This is the subset of $X$ where $\operatorname{codim} X_{k} \geq k+2, k \geq p+1$, cf., Sect. 6. Thus it contains all points $x$ such that $\mathscr{O}_{X, x}$ is Cohen-Macaulay. In particular, (7.3) is an isomorphism in $X_{\text {reg }}$.

Theorem 7.3 is a consequence of the results in [22]. If $X$ has pure dimension $p$, there is an injective mapping

$$
\mathscr{O}_{X} \rightarrow \mathcal{H o m}\left(\mathcal{E}_{x t^{p}}\left(\mathscr{O}_{X}, K_{\Omega}\right), \mathcal{C H} \mathcal{H}_{\Omega}^{Z}\right)
$$

which by [22, Theorem 1.2 and Remark 6.11] is an isomorphism if and only if $\mathscr{O}_{X}$ is $S_{2}$. Since the image of such a morphism must be annihilated by $\mathcal{J}$ by linearity, it is indeed a morphism

$$
\mathscr{O}_{X} \rightarrow \mathcal{H o m}\left(\mathcal{E x t}^{p}\left(\mathscr{O}_{X}, K_{\Omega}\right), \mathcal{H o m}\left(\mathscr{O}_{\Omega} / \mathcal{J}, \mathcal{C H}_{\Omega}^{Z}\right)\right)
$$

In view of (4.2) and (5.3), (7.5) corresponds to a morphism $\mathscr{O}_{X} \rightarrow \mathcal{H o m}\left(\omega_{X}^{n}, \omega_{X}^{n}\right)$, and the fact that it is the morphism (7.3) is a rather simple consequence of the definition of the morphism (7.4) in [22, (6.9)].

As mentioned in the introduction, Theorem 7.3 can be seen as a reformulation of a classical result of Roos, [30], which is the same statement about the injection

$$
\mathscr{O}_{\Omega} / \mathcal{J} \rightarrow \mathcal{E} x t^{p}\left(\mathcal{E} x t^{p}\left(\mathscr{O}_{\Omega} / \mathcal{J}, K_{\Omega}\right), K_{\Omega}\right)
$$

here we assume that the ideal has pure dimension. The equivalence of the morphisms (7.4) and (7.6) is discussed in [22, Corollary 1.4].

Let us now consider the case when $X$ is reduced. Since sections of $\omega_{X}^{n}$ are meromorphic, see [6, Example 2.8], and thus almost semi-meromorphic and generically smooth, by Proposition 2.4 (with $Z=X=\Omega$ ) we can extend (7.3) to a morphism

$$
\mathcal{W}_{X}^{0, *} \rightarrow \mathcal{H o m}\left(\omega_{X}^{n}, \mathcal{W}_{X}^{n, *}\right)
$$


Lemma 7.4 When $X$ is reduced (7.7) is an isomorphism.

Thus Definition 7.2 is consistent with the previous definition of $\mathcal{W}_{X}^{0 \text {,* }}$ when $X$ is reduced.

Proof Clearly each $\phi$ in $\mathcal{W}_{X}^{0, *}$ defines an element $\alpha$ in $\mathcal{H o m}\left(\omega_{X}^{n}, \mathcal{W}_{X}^{n, *}\right)$ by $h \mapsto \phi \wedge h$. If we apply this to a generically nonvanishing $h$ we see by the SEP that (7.7) is injective.

For the surjectivity, take $\alpha$ in $\mathcal{H o m}\left(\omega_{X}^{n}, \mathcal{W}_{X}^{n, *}\right)$. If $h^{\prime}$ is nonvanishing at a point on $X_{\text {reg }}$, then it generates $\omega_{X}^{n}$ and thus $\alpha$ is determined by $\phi:=\alpha h^{\prime}$ there. By [10, Theorem 3.7], $\phi=\psi \wedge h^{\prime}$ for a unique current $\psi$ in $\mathcal{W}_{X}^{0, *}$ so by $\mathscr{O}_{X}$-linearity $\alpha h=\psi \wedge h$ for any $h$. Hence, $\psi$ is well-defined as a current in $\mathcal{W}_{X}^{0, *}$ on $X_{\text {reg }}$.

We must verify that $\psi$ has an extension in $\mathcal{W}_{X}^{0, *}$ across $X_{\text {sing. }}$. Since such an extension must be unique by the SEP, the statement is local on $X$. Thus we may assume that $\alpha$ is defined on the whole of $X$ and that there is a generically nonvanishing holomorphic $n$-form $\gamma$ on $X$. Then $\alpha \gamma$ is a section of $\mathcal{W}^{n, *}(X)$.

Let us choose a smooth modification $\pi: X^{\prime} \rightarrow X$ that is biholomorphic outside $X_{\text {sing. }}$. Then $\pi^{*} \gamma$ is a holomorphic $n$-form on $X^{\prime}$ that is generically non-vanishing. We claim that there is a current $\tau$ in $\mathcal{W}^{n, 0}\left(X^{\prime}\right)$ such that $\pi_{*} \tau=\alpha \gamma$. In fact, $\tau$ exists on $\pi^{-1}\left(X_{\text {reg }}\right)$ since $\pi$ is a biholomorphism there. Moreover, by [4, Proposition 1.2], $\alpha h$ is the direct image of some pseudomeromorphic current $\tilde{\tau}$ on $X^{\prime}$, and is therefore also the image of the (unique) current $\tau=\mathbf{1}_{\pi^{-1}\left(X_{\text {reg }}\right)} \tilde{\tau}$ in $\mathcal{W}^{n, *}\left(X^{\prime}\right)$.

By [10, Theorem 3.7] again $\tau$ is locally of the form $\xi \wedge d s$, where $\xi$ is in $\mathcal{W}_{X^{\prime}}^{0, *}$ and $d s=d s_{1} \wedge \cdots \wedge d s_{n}$ for some local coordinates $s$. Hence, $\tau$ is a $K_{X^{\prime}}$-valued section of $\mathcal{W}^{0, *}\left(X^{\prime}\right)$, so $\tau / \pi^{*} \gamma$ is a section of $\mathcal{W}^{0, *}\left(X^{\prime}\right)$. Now $\Psi:=\pi_{*}\left(\tau / \pi^{*} \gamma\right)$ is a section of $\mathcal{W}^{0, *}(X)$. On $X_{\text {reg }} \cap\{\gamma \neq 0\}$ we thus have that $\Psi \wedge \gamma=\pi_{*} \tau=\alpha \gamma=\psi \wedge \gamma$ and so $\Psi=\psi$ there. By the SEP it follows that $\Psi$ coincides with $\psi$ on $X_{\text {reg }}$ and is thus the desired pseudomeromorphic extension to $X$.

In view of (5.1) and (5.3) we have, given a local embedding $i: X \rightarrow \Omega$, the extrinsic representation

$\mathcal{W}_{X}^{0, *} \simeq \mathcal{H o m}\left(\mathcal{H o m}\left(\mathscr{O}_{\Omega} / \mathcal{J}, \mathcal{C H}_{\Omega}^{Z}\right), \mathcal{H o m}\left(\mathscr{O}_{\Omega} / \mathcal{J}, \mathcal{W}_{\Omega}^{Z}\right)\right), \phi \mapsto\left(i_{*} h \mapsto i_{*}(\phi \wedge h)\right)$

Lemma 7.5 Assume that $X_{\text {reg }} \rightarrow \Omega$ is a local embedding and $(z, w)$ coordinates as before. Each section $\phi$ in $\mathcal{W}_{X}^{0, *}$ has a unique representation (7.1) with $\widehat{\phi}_{j}$ in $\mathcal{W}_{Z}^{0, *}$.

A current with a representation (7.1) is considered as an element of $\mathcal{W}_{X}^{0, *}=$ $\mathcal{H}$ om $\left(\omega_{X}^{n}, \mathcal{W}_{X}^{n, *}\right)$ in view of the comment after Lemma 7.1 .

Proof From (4.9) we get an induced sequence

$$
0 \rightarrow\left(\mathcal{W}_{Z}^{0, *}\right)^{\mathcal{v}} \stackrel{T}{\rightarrow}\left(\mathcal{W}_{Z}^{0, *}\right)^{M} \stackrel{A}{\rightarrow}\left(\mathcal{W}_{Z}^{0, *}\right)^{M^{\prime}}
$$

which is also exact. In fact, $T$ in (7.9) is clearly injective, and by $(4.10)$, if $\xi$ in $\left(\mathcal{W}_{Z}^{0, *}\right)^{M}$ and $A \xi=0$, then $T \eta=\xi$, if $\eta=S \xi$. 
Now take $\phi$ in $\mathcal{H}$ om $\left(\omega_{X}^{n}, \mathcal{W}_{X}^{n, *}\right)$. Let us choose a basis $\mu^{1}, \ldots, \mu^{m}$ for $\omega_{X}^{n}$ and let $\tilde{\phi}$ be the element in $\left(\mathcal{W}_{Z}^{0, *}\right)^{M}$ obtained from the coefficients of $\phi \mu^{j}$ when expressed as in (2.11), cf., Sect. 4.1. We claim that $A \tilde{\phi}=0$. Taking this for granted, by the exactness of (7.9), $\tilde{\phi}$ is the image of the tuple $\hat{\phi}=S \tilde{\phi}$. Now $\hat{\phi} \wedge \mu^{j}=\phi \mu^{j}$ since they are represented by the same tuple in $\left(\mathcal{W}_{Z}^{0, *}\right)^{M}$. Thus $\hat{\phi}$ gives the desired representation of $\phi$.

In view of Proposition 2.9 it is enough to prove the claim where $\tilde{\phi}$ is smooth. Let us therefore fix such a point, say 0 , and show that $(A \tilde{\phi})(0)=0$. From the proof of Lemma 4.11, if we let $\mathcal{I}$ be the ideal generated by $z$, and let $X_{0}$ be defined by $\mathscr{O}_{X_{0}}:=\mathscr{O}_{\Omega} /(\mathcal{J}+\mathcal{I})$, then $\mu^{1} \wedge \mu^{z}, \ldots, \mu^{m} \wedge \mu^{z}$ generate $\omega_{X_{0}}^{0}$. If we let $\phi_{0}$ be the morphism in $\mathcal{H o m}\left(\omega_{X_{0}}^{0}, \omega_{X_{0}}^{0}\right)$ given by $\phi_{0}\left(\mu^{i} \wedge \mu^{z}\right):=\phi \mu^{i} \wedge \mu^{z}$ (which indeed gives a well-defined such morphism), then, as in the proof of Lemma 4.11, $\tilde{\phi}_{0}=\tilde{\phi}(0)$. In addition, the sequence (4.9) for $X_{0}$ is

$$
0 \rightarrow \mathbb{C}^{\nu} \stackrel{T(0)}{\rightarrow} \mathbb{C}^{M} \stackrel{A(0)}{\rightarrow} \mathbb{C}^{M^{\prime}}
$$

Since $X_{0}$ is 0-dimensional, the morphism $\mathscr{O}_{X_{0}} \rightarrow \mathcal{H o m}\left(\omega_{X_{0}}, \omega_{X_{0}}\right)$ is an isomorphism by Theorem 7.3, and thus $\phi_{0}$ is given as multiplication by a function in $\mathscr{O}_{X_{0}}$, which we also denote by $\phi_{0}$, i.e., $\tilde{\phi}_{0}=T(0) \hat{\phi}_{0}$. Hence, $A(0) \tilde{\phi}_{0}=A(0) T(0) \hat{\phi}_{0}=0$, and thus $(A \tilde{\phi})(0)=0$.

Example 7.6 (Meromorphic functions) Assume that we have a local embedding $X \rightarrow$ $\Omega$. Given meromorphic functions $\Phi, \Phi^{\prime}$ in $\Omega$ that are holomorphic generically on $Z$, we say that $\Phi \sim \Phi^{\prime}$ if and only if $\Phi-\Phi^{\prime}$ is in $\mathcal{J}$ generically on $Z$. If $\Phi=A / B$ and $\Phi^{\prime}=A^{\prime} / B^{\prime}$, where $B$ and $B^{\prime}$ are generically non-vanishing on $Z$, the condition is precisely that $A B^{\prime}-A^{\prime} B$ is in $\mathcal{J}$. We say that such an equivalence class is a meromorphic function $\phi$ on $X$, i.e., $\phi$ is in $\mathcal{M}_{X}$. Clearly we have $\mathscr{O}_{X} \subset \mathcal{M}_{X}$. We claim that

$$
\mathcal{M}_{X} \subset \mathcal{W}_{X}^{0, *}
$$

To see this, first notice that if we take a representative $\Phi$ in $\mathcal{M}_{\Omega}$ of $\phi$, then it can be considered as an almost semi-meromorphic current on $\Omega$ with Zariski-singular support of positive codimension on $Z$, since it is generically holomorphic on $Z$. As in Definition 5.5 we therefore have a current $\Phi \wedge h$ in $\mathcal{W}_{X}^{n, 0}$ for $h$ in $\omega_{X}^{n}$. Another representative $\Phi^{\prime}$ of $\phi$ will give rise to the same current generically and hence everywhere by the SEP. Thus $\phi$ defines a section of $\mathcal{H o m}\left(\omega_{X}^{n}, \mathcal{W}_{X}^{n, *}\right)=\mathcal{W}_{X}^{0, *}$.

By definition, a current $\phi$ in $\mathcal{W}_{X}^{0, *}$ can be multiplied by a current $h$ in $\omega_{X}^{n}$, and the product $\phi \wedge h$ lies in $\mathcal{W}_{X}^{n, *}$. It will be crucial that we can extend to products by somewhat more general currents. Notice that $\omega_{X}^{n}$ is a subsheaf of $\mathcal{C}_{X}^{n \text {,* }}$, which is an $\mathscr{E}_{X}^{0, *}$-module. Thus, we can consider the subsheaf $\mathscr{E}_{X}^{0, *} \omega_{X}^{n}$ of $\mathcal{C}_{X}^{n, *}$ which consists of finite sums $\sum \xi_{i} \wedge h_{i}$, where $\xi_{i}$ are in $\mathscr{E}_{X}^{0, *}$ and $h_{i}$ are in $\omega_{X}^{n}$. 
Lemma 7.7 Each $\phi$ in $\mathcal{W}_{X}^{0, *}=\mathcal{H}_{\mathscr{O}_{X}}\left(\omega_{X}^{n}, \mathcal{W}_{X}^{n, *}\right)$ has a unique extension to a morphism in $\mathcal{H o m}_{\mathscr{E}_{X}^{0, *}}\left(\mathscr{E}_{X}^{0, *} \omega_{X}^{n}, \mathcal{W}_{X}^{n, *}\right)$.

Proof The uniqueness follows by $\mathscr{E}_{X}^{0, *}$-linearity, i.e., if $b=\xi_{1} \wedge h_{1}+\cdots+\xi_{r} \wedge h_{r}$ is in $\mathscr{E}_{X}^{0, *} \omega_{X}^{n}$, then one must have

$$
\phi b=\sum_{i}(-1)^{\left(\operatorname{deg} \xi_{i}\right)(\operatorname{deg} \phi)} \xi_{i} \wedge \phi h_{i} .
$$

We must check that this is well-defined, i.e., that the right hand side does not depend on the representation $\xi_{1} \wedge h_{1}+\cdots+\xi_{r} \wedge h_{r}$ of $b$. By the SEP, it is enough to prove this locally on $X_{\text {reg, }}$, and we can then assume that $\phi$ has a representation (7.1). By Proposition 2.9, it is then enough to prove that it is well-defined assuming that $\widehat{\phi}_{0}, \ldots, \widehat{\phi}_{\nu-1}$ in (7.1) are all smooth. In this case, the right hand side of (7.10) is simply the product of $\xi_{1} \wedge h_{1}+\cdots+\xi_{r} \wedge h_{r}=b$ by the smooth form $\phi$ in $\mathscr{E}_{X}^{0}$,* and this product only depends on $b$.

Corollary 7.8 Let $\phi$ be a current in $\mathcal{W}_{X}^{0, *}$ and let $\alpha$ be a current in $\mathcal{W}_{X}^{n, *}$ of the form $\alpha=\sum a_{i} \wedge h_{i}$, where $a_{i}$ are almost semi-meromorphic $(0, *)$-currents on $\Omega$ which are generically smooth on $Z$, and $h_{i}$ are in $\omega_{X}^{n}$. Then one has a well-defined product

$$
\phi \wedge \alpha=\sum(-1)^{\left(\operatorname{deg} a_{i}\right)(\operatorname{deg} \phi)} a_{i} \wedge\left(\phi \wedge h_{i}\right)
$$

Proof The right hand side of (7.11) exists as a current in $\mathcal{W}_{X}^{n, *}$, and we must prove is that it only depends on the current $\alpha$ and not on the representation $\sum a_{i} \wedge h_{i}$. Notice that all the $a_{i}$ are smooth outside some subvariety $V$ of $Z$ and there the right hand side of (7.11) is the product of $\phi$ and $\alpha$ in $\mathscr{E}_{X}^{0, *} \omega_{X}^{n}$, cf., Lemma 7.7. It follows by the SEP that the right hand side only depends on $\alpha$.

Remark 7.9 Recall from (6.9) that $\omega=b \vartheta$. If $\phi$ is in $\mathcal{W}_{X}^{0, *}$, then we can define the product $\phi \wedge \omega$ by Corollary 7.8.

Expressed extrinsically, if $\mu=i_{*} \vartheta$, and if we write $R \wedge d z=b \mu$ as in Lemma 6.2, then we can define the product $R \wedge d z \wedge \phi:=b \mu \wedge \phi$ as a current in $\mathcal{H o m}\left(\mathscr{O}_{\Omega} / \mathcal{J}, \mathcal{W}_{\Omega}^{Z}\right)$.

Lemma 7.10 Assume that $\phi$ is in $\mathcal{W}_{X}^{0, *}$, and that $\phi \wedge \omega=0$ for some structure form $\omega$, where the product is defined by Remark 7.9. Then $\phi=0$.

Proof Considering the component with values in $E_{p}$, we get that $\phi \wedge \omega_{0}=0$. By Proposition 6.7, any $h$ in $\omega_{X}^{n}$ can be written as $h=\xi \omega_{0}$, where $\xi$ is a holomorphic section of $E_{p}^{*}$, so by $\mathscr{O}$-linearity, $\phi \wedge h=0$, i.e., $\phi=0$.

We end this section with the following result, first part of [10, Theorem 3.7]. We include here a different proof than the one in [10], since we believe the proof here is instructive. 
Proposition 7.11 If $Z$ is smooth, then $\mathcal{W}_{Z}$ is closed under holomorphic differential operators.

Proof Let $\tau$ be any current in $\mathcal{W}_{Z}$. It suffices to prove that if $\zeta$ are local coordinates on $Z$, then $\partial \tau / \partial \zeta_{1}$ is in $\mathcal{W}_{Z}$. Consider the current

$$
\tau^{\prime}=\tau \otimes \bar{\partial} \frac{d w}{2 \pi i w^{2}}
$$

on the manifold $Y:=Z \times \mathbb{C}_{w}$. Clearly $\tau^{\prime}$ has support on $Z$, and it follows from (2.5) that $\tau^{\prime}$ is in $\mathcal{W}_{Y}^{Z}$. Let

$$
p:(z, w) \mapsto \zeta=\left(z_{1}+w, z_{2}, \ldots, z_{n}\right),
$$

which is just a change of variables on $Y$ followed by a projection. It follows from (2.4) that $p_{*} \tau^{\prime}$ is in $\mathcal{W}_{Z}$. Since

$$
\bar{\partial} \frac{d w}{2 \pi i w^{2}} \cdot \xi(w)=\frac{\partial \xi}{\partial w}(0)
$$

it is readily verified that $p_{*} \tau^{\prime}=\partial \tau / \partial \zeta_{1}$, so we conclude that $\partial \tau / \partial \zeta_{1}$ is in $\mathcal{W}_{Z}$.

\section{The $\bar{\partial}$-operator on $\mathcal{W}_{X}^{0, *}$}

We already know the meaning of $\bar{\partial}$ on $\mathcal{W}_{X}^{n, *}$, and we now define $\bar{\partial}$ on $\mathcal{W}_{X}^{0, *}$.

Definition 8.1 Assume that $\phi, v$ are in $\mathcal{W}_{X}^{0, *}$, We say that $\bar{\partial} v=\phi$ if

$$
\bar{\partial}(v \wedge h)=\phi \wedge h, \quad h \in \omega_{X}^{n}
$$

If we have an embedding $X \rightarrow \Omega$, (8.1) means, cf., (7.8), that

$$
\bar{\partial}(v \wedge \mu)=\phi \wedge \mu, \quad \mu \in \mathcal{H} \text { om }\left(\mathscr{O}_{\Omega} / \mathcal{J}, \mathcal{C H}_{\Omega}^{Z}\right)
$$

In view of Remark 7.9 we can define the product $\phi \wedge \omega$ for $\phi$ in $\mathcal{W}_{X}^{0, *}$.

Definition 8.2 We say that $v$ belongs to Dom $\bar{\partial}_{X}$ if $v$ is in Dom $\bar{\partial}$, i.e., $\bar{\partial} v=\phi$ for some $\phi$ and in addition $\bar{\partial}(v \wedge \omega)$, a priori only in $\mathcal{P} \mathcal{M}_{X}^{n, *}$, is in $\mathcal{W}_{X}^{n, *}$, for each structure form $\omega$ from any possible embedding.

If $X$ is Cohen-Macaulay, then any such $\omega$ is of the form $a_{1} h^{1}+\cdots+a_{m} h^{m}$, where $h^{j}$ are in $\omega_{X}^{n}$ and $a_{j}$ are smooth, see Remark 6.5, and hence Dom $\bar{\partial}_{X}$ coincides with Dom $\bar{\partial}$ in this case. 
Example 8.3 Assume that $v$ is in $\mathscr{E}_{X}^{0, *}$ and $\phi=\bar{\partial} v$ in the sense in Section 4. Then clearly

$$
\bar{\partial}(v \wedge \omega)=\phi \wedge \omega+(-1)^{\operatorname{deg} v} v \wedge \bar{\partial} \omega
$$

Since $\bar{\partial} \omega=f \omega$, and $\mathcal{W}_{X}^{n, *}$ is closed under multiplication with forms in $\mathscr{E}_{X}^{0, *}$, we get that $\bar{\partial}(v \wedge \omega)$ is in $\mathcal{W}_{X}^{n, *}$, so $v$ is in Dom $\bar{\partial}_{X}$ and $\bar{\partial}_{X} v=\phi$.

If $w$ is in Dom $\bar{\partial}_{X}$ and $v$ is in $\mathscr{E}_{X}^{0, *}$, then

$$
\bar{\partial}(v \wedge w \wedge \omega)=\bar{\partial} v \wedge w \wedge \omega+(-1)^{\operatorname{deg} v} v \wedge \bar{\partial}(w \wedge \omega)
$$

Thus $v \wedge w$ is in Dom $\bar{\partial}_{X}$, and the Leibniz rule $\bar{\partial}(v \wedge w)=\bar{\partial} v \wedge w+(-1)^{\operatorname{deg} v} v \wedge \bar{\partial} w$ holds.

Let $\chi_{\delta}=\chi\left(|h|^{2} / \delta\right)$ where $h$ is a tuple of holomorphic functions that cuts out $X_{\text {sing }}$.

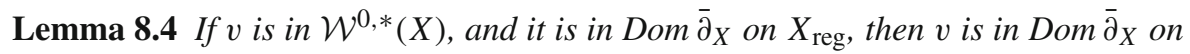
all of $X$ if and only if

$$
\bar{\partial} \chi_{\delta} \wedge v \wedge \omega \rightarrow 0, \quad \delta \rightarrow 0,
$$

for all structure forms $\omega$. In this case,

$$
-\nabla_{f}(v \wedge \omega)=\bar{\partial} v \wedge \omega
$$

Proof Since $\mathcal{W}_{X}^{n, *}$ is closed under multiplication by $f, v$ is in Dom $\bar{\partial}_{X}$ if and only if $\nabla_{f}(v \wedge \omega)$ is in $\mathcal{W}_{X}^{n, *}$ for all structure forms $\omega$. Since $v$ is in Dom $\bar{\partial}_{X}$ on $X_{\text {reg }}$, thus $\nabla_{f}(v \wedge \omega)$ is in $\mathcal{W}_{X}^{n, *}$ on $X_{\text {reg. }}$ By (2.2), $\nabla_{f}(v \wedge \omega)$ is then in $\mathcal{W}_{X}^{n, *}$ on all of $X$ if and only if

$$
\mathbf{1}_{X_{\mathrm{reg}}} \nabla_{f}(v \wedge \omega)=\nabla_{f}(v \wedge \omega) .
$$

By the Leibniz rule,

$$
\nabla_{f}\left(\chi_{\delta} v \wedge \omega\right)=-\bar{\partial} \chi_{\delta} \wedge v \wedge \omega+\chi_{\delta} \nabla_{f}(v \wedge \omega)
$$

Since $v$ is in $\mathcal{W}_{X}^{0, *}, v \wedge \omega$ is in $\mathcal{W}_{X}^{n, *}$, so the left hand side of (8.6) tends to $\nabla_{f}(v \wedge \omega)$ when $\delta \rightarrow 0$, whereas the second term on the right hand side of (8.6) tends to $\mathbf{1}_{X_{\text {reg }}} \nabla_{f}(v \wedge \omega)$. Thus (8.5) holds if and only if (8.3) does. Thus the first statement in the lemma is proved.

Recall, cf., (6.9), that $\omega=b \vartheta$ where $b$ is smooth on $X_{\text {reg }}$ and $\vartheta$ is in $\omega_{X}^{n}$. By the Leibniz rule thus $-\nabla_{f}(v \wedge \omega)=\bar{\partial} v \wedge \omega$ on $X_{\text {reg }}$, since $\nabla_{f} \omega=0$. Therefore, (8.6) is equivalent to $-\nabla_{f}\left(\chi_{\delta} v \wedge \omega\right)=\bar{\partial} \chi_{\delta} \wedge v \wedge \omega+\chi_{\delta} \bar{\partial} v \wedge \omega$. If (8.3) holds, we therefore get (8.4) when $\delta \rightarrow 0$.

Remark 8.5 In case $X$ is reduced the definition of $\bar{\partial}_{X}$ is precisely the same as in [6]. However, the definition of $\bar{\partial} v=\phi$ given here, for $v, \phi$ in $\mathcal{W}_{X}^{0, *}$, does not coincide with the definition in, e.g., [6]. In fact, that definition means that $\bar{\partial}(v \wedge h)=\phi \wedge h$ for all smooth $h$ in $\omega_{X}^{n}$, which in general is a strictly weaker condition. For example, for 
any weakly holomorphic function $v$, we have $\bar{\partial}(v \wedge h)=0$ for all smooth $h$ in $\omega_{X}^{n}$, while if $X$ is a reduced complete intersection, or more generally Cohen-Macaulay, then $\bar{\partial}(v \wedge h)=0$ for all $h$ in $\omega_{X}^{n}$ is equivalent to $v$ being strongly holomorphic, see $[33$, p. 124] and [2].

We conclude this section with a lemma that shows that $\bar{\partial}$ means what one should expect when $\phi, v$ are expressed with respect to a local basis $w^{\alpha_{j}}$ for $\mathscr{O}_{X}$ over $\mathscr{O}_{Z}$ as in Lemma 7.5.

Lemma 8.6 Assume that we have a local embedding $X_{\text {reg }} \rightarrow \Omega$ and $\phi, v$ in $\mathcal{W}_{X}^{0, *}$ represented as in (7.1). Then $\bar{\partial} v=\phi$ if and only if

$$
\bar{\partial} \hat{v}_{j}=\hat{\phi}_{j}, \quad j=0, \ldots, v-1 .
$$

Proof Let us use the notation from the proof of Lemma 7.5. Recall that $\hat{v}=S \tilde{v}$. In view of (8.2) and (2.12), $\widetilde{\partial} v=\bar{\partial} \tilde{v}$. Since $S$ is holomorphic therefore $\widehat{\bar{\partial} v}=S \widetilde{\tilde{\partial} v}=$ $S \bar{\partial} \tilde{v}=\bar{\partial}(S \tilde{v})=\bar{\partial} \hat{v}$.

\section{Solving $\bar{\partial} u=\phi$ on $X$}

We will find local solutions to the $\bar{\partial}$-equation on $X$ by means of integral formulas. We use the notation and machinery from [6, Section 5]. Let $i: X \rightarrow \Omega \subset \mathbb{C}^{N}$ be a local embedding such that $\Omega$ is pseudoconvex, let $\Omega^{\prime} \subset \subset \Omega$ be a relatively compact subdomain of $\Omega$, and let $X^{\prime}=X \cap \Omega^{\prime}$.

Theorem 9.1 There are integral operators

$$
K: \mathscr{E}^{0, *+1}(X) \rightarrow \mathcal{W}^{0, *}\left(X^{\prime}\right) \cap \operatorname{Dom} \bar{\partial}_{X}, \quad P: \mathscr{E}^{0, *}(X) \rightarrow \mathscr{E}^{0, *}\left(X^{\prime}\right)
$$

such that, for $\phi \in \mathscr{E}^{0, k}(X)$,

$$
\phi=\bar{\partial} K \phi+K(\bar{\partial} \phi)+P \phi .
$$

The operators $K$ and $P$ are described below; they depend on a choice of weight $g$. Since $\Omega$ is Stein one can find such a weight $g$ that is holomorphic in $z$, by which we mean that it depends holomorphically on $z \in \Omega^{\prime}$ and has no components containing any $d \bar{z}_{i}$, cf., Example 5.1 in [6]. In this case, $P \phi$ is holomorphic when $k=0$, and vanishes when $k \geq 1$, i.e.,

$$
\phi=\bar{\partial} K \phi+K(\bar{\partial} \phi), \quad \phi \in \mathscr{E}^{0, k}(X), \quad k \geq 1 .
$$

If $\bar{\partial} \phi=0$ in $\Omega$, and $k \geq 1$, then $K \phi$ is a solution to $\bar{\partial} v=\phi$. If $k=0$, then $\phi=P \phi$ is holomorphic. It follows that a smooth $\bar{\partial}$-closed function is holomorphic. In the reduced case this is a classical theorem of Malgrange [24]. In Sect. 10 we prove that $K \phi$ is smooth on $X_{\text {reg. }}$. 
We now turn to the definition of $K$ and $P$. For future need, in Sect. 11, we define them acting on currents in $\mathcal{W}^{0, *}(X)$ and not only on smooth forms. Let $\pi: \Omega_{\zeta} \times \Omega_{z}^{\prime} \rightarrow \Omega_{z}^{\prime}$ be the natural projection. Let us choose a holomorphic Hefer form ${ }^{3} H$, a smooth weight $g$ with compact support in $\Omega$ with respect to $z \in \Omega^{\prime} \subset \subset \Omega$, and let $B$ be the Bochner-Martinelli form. Since we are only are concerned with $(0, *)$-forms, we will here assume that $H$ and $B$ only have holomorphic differentials in $\zeta$, i.e., the factors $d \eta_{i}=d \zeta_{i}-d z_{i}$ in $H$ and $B$ in [6] should be replaced by just $d \zeta_{i}$.

If $\gamma$ is a current in $\Omega_{\zeta} \times \Omega_{z}^{\prime}$ we let $(\gamma)_{N}$ be the component of bidegree $(N, *)$ in $\zeta$ and $(0, *)$ in $z$, and let $\vartheta(\gamma)$ be the current such that

$$
\vartheta(\gamma) \wedge d \zeta=(\gamma)_{N}
$$

Consider now $\mu$ in $\mathcal{H o m}\left(\mathscr{O}_{\Omega} / \mathcal{J}, \mathcal{W}_{\Omega}^{Z}\right)$ and $\phi$ in $\mathcal{W}_{X}^{0, *}$. We can give meaning to

$$
(g \wedge H R(\zeta))_{N} \wedge \phi(\zeta) \wedge \mu(z)
$$

as a tensor product of currents in the following way: first of all, by Remark 7.9, we can form the product $R(\zeta) \wedge d \zeta \wedge \phi(\zeta)$ as a current in $\mathcal{W}_{\Omega}^{Z}$. In view of [11, Corollary 4.7] the tensor product $R(\zeta) \wedge d \zeta \wedge \phi(\zeta) \wedge \mu(z)$ is in $\mathcal{W}_{\Omega_{\zeta} \times \Omega_{z}^{\prime}}^{Z \times Z^{\prime}}$, where $Z^{\prime}=Z \cap \Omega^{\prime}$. Finally, we multiply this with the smooth form $\vartheta(g \wedge H)$ to obtain (9.4). Similarly, outside of $\Delta$, the diagonal in $\Omega \times \Omega^{\prime}$, where $B$ is smooth, we can define

$$
(B \wedge g \wedge H R(\zeta))_{N} \wedge \phi(\zeta) \wedge \mu(z)
$$

as a tensor product of currents.

Lemma 9.2 For $\mu$ in $\mathcal{H o m}\left(\mathscr{O}_{\Omega^{\prime}} / \mathcal{J}, \mathcal{W}_{\Omega^{\prime}}^{Z^{\prime}}\right)$ and $\phi \in \mathcal{W}^{0, *}(X)$, the current (9.5), a priori defined as a current in $\mathcal{W}_{\Omega_{\zeta} \times \Omega_{z}^{\prime} \backslash \Delta}^{Z \times Z_{\Delta}^{\prime} \backslash \Delta}$ has an extension across $\Delta$. The current (9.4) and the extension of (9.5) depend $\mathscr{O}_{\Omega} / \mathcal{J}$-bilinearly on $\mu$ and $\phi$, and are such that

$$
K \phi \wedge \mu:=\pi_{*}\left((B \wedge g \wedge H R(\zeta))_{N} \wedge \phi(\zeta) \wedge \mu(z)\right)
$$

and

$$
P \phi \wedge \mu:=\pi_{*}\left((g \wedge H R(\zeta))_{N} \wedge \phi(\zeta) \wedge \mu(z)\right)
$$

are in $\mathcal{H o m}\left(\mathscr{O}_{\Omega^{\prime}} / \mathcal{J}, \mathcal{W}_{\Omega^{\prime}}^{Z^{\prime}}\right)$.

It follows that $K \phi \wedge \mu$ and $P \phi \wedge \mu$ are $\mathbb{C}$-linear in $\phi$ and $\mathscr{O}_{\Omega^{\prime}} / \mathcal{J}$-linear in $\mu$. In view of (7.8), by considering $\mu$ in $\mathcal{H o m}\left(\mathscr{O}_{\Omega^{\prime}} / \mathcal{J}, \mathcal{C} \mathcal{H}_{\Omega^{\prime}}^{Z^{\prime}}\right)$, we have defined linear operators

$$
K: \mathcal{W}^{0, *+1}(X) \rightarrow \mathcal{W}^{0, *}\left(X^{\prime}\right), \quad P: \mathcal{W}^{0, *}(X) \rightarrow \mathcal{W}^{0, *}\left(X^{\prime}\right) .
$$

Proof of Lemma 9.2 In order to define the extension of (9.5) across $\Delta$, we note first that since $B$ is almost semi-meromorphic with Zariski singular support $\Delta, \vartheta(B \wedge g \wedge H)$

\footnotetext{
3 We are only concerned with the component $H^{0}$ of this form, so for simplicity we write just $H$.
} 
is an almost semi-meromorphic $(0, *)$-current on $\Omega_{\zeta} \times \Omega_{z}^{\prime}$, which is smooth outside the diagonal. We can thus form the current $\vartheta(B \wedge g \wedge H) \wedge R(\zeta) \wedge d \zeta \wedge \phi(\zeta) \wedge \mu(z)$ in $\mathcal{W}_{\Omega_{\zeta} \times \Omega_{z}^{\prime}}^{Z \times Z^{\prime}}$, cf., Proposition 2.4, and this is the extension of (9.5) across $\Delta$.

From the definitions above, it is clear that (9.4) and the extension of (9.5) are $\mathscr{O}_{\Omega^{-}}$ bilinear in $\phi$ and $\mu$. Both these currents are annihilated by $\mathcal{J}_{z}$ and $\mathcal{J}_{\zeta}$, cf., (2.8), so they depend $\mathscr{O}_{\Omega} / \mathcal{J}$-bilinearly. In view of (2.4) we conclude that (9.6) and (9.7) are in $\mathcal{H}$ om $\left(\mathscr{O}_{\Omega^{\prime}} / \mathcal{J}, \mathcal{W}_{\Omega^{\prime}}^{Z^{\prime}}\right)$.

Proposition 9.3 If $\phi \in \mathcal{W}^{0, k}(X)$, then $P \phi \in \mathscr{E}^{0, k}\left(X^{\prime}\right)$, and if in addition $g$ is holomorphic in $z$, then $P \phi \in \mathscr{O}\left(X^{\prime}\right)$ if $k=0$ and vanishes if $k \geq 1$.

Proof Since $\vartheta(g \wedge H)$ is smooth, we get that

$$
\begin{aligned}
& \pi_{*}(\vartheta(g \wedge H) \wedge R(\zeta) \wedge d \zeta \wedge \phi \wedge \mu(z)) \\
& \quad=\pi_{*}(\vartheta(g \wedge H) \wedge R(\zeta) \wedge d \zeta \wedge \phi) \wedge \mu(z)=\pi_{*}\left((g \wedge H R)_{N} \wedge \phi\right) \wedge \mu(z)
\end{aligned}
$$

cf., for example [20, (5.1.2)]. Thus $P \phi(z)=\pi_{*}\left((g \wedge H R(\zeta))_{N} \wedge \phi\right)$ which is smooth on $\Omega^{\prime}$. If $g$ depends holomorphically on $z$, then $P \phi$ is holomorphic in $\Omega^{\prime}$ if $\phi$ is a $(0,0)$-current, and vanishes for degree reasons if $\phi$ has positive degree.

We shall now approximate $K \phi$ by smooth forms. Let $B^{\epsilon}=\chi\left(|\zeta-z|^{2} / \epsilon\right) B$.

Proposition 9.4 For any $\phi \in \mathcal{W}^{0, k}(X), k \geq 1$,

$$
K^{\epsilon} \phi:=\pi_{*}\left(\left(B^{\epsilon} \wedge g \wedge H R(\zeta)\right)_{N} \wedge \phi\right)=\pi_{*}\left(\vartheta\left(B^{\epsilon} \wedge g \wedge H\right) \wedge R(\zeta) \wedge d \zeta \wedge \phi\right)
$$

is in $\mathscr{E}^{0, k-1}\left(X^{\prime}\right)$ and $K^{\epsilon} \phi \rightarrow K \phi$ when $\epsilon \rightarrow 0$.

The last statement means that

$$
K^{\epsilon} \phi \wedge \mu \rightarrow K \phi \wedge \mu, \quad \mu \in \mathcal{H o m}\left(\mathscr{O}_{\Omega^{\prime}} / \mathcal{J}, \mathcal{C H}_{\Omega^{\prime}}^{Z^{\prime}}\right)
$$

Proof Since $B^{\epsilon}$ is smooth, the current we push forward is $R(\zeta) \wedge \phi(\zeta)$ times a smooth form of $\zeta$ and $z$. Therefore $K^{\epsilon} \phi$ is smooth. As in the proof of Proposition 9.3, we obtain since $B^{\epsilon}$ is smooth that

$$
K^{\epsilon} \phi \wedge \mu=\pi_{*}\left(\left(B^{\epsilon} \wedge g \wedge H R(\zeta)\right)_{N} \wedge \phi \wedge \mu(z)\right)
$$

By (5.2) applied to $a=B$ we have that

$$
\left(B^{\epsilon} \wedge g \wedge H R(\zeta)\right)_{N} \wedge \phi \wedge \mu(z) \rightarrow(B \wedge g \wedge H R(\zeta))_{N} \wedge \phi \wedge \mu(z)
$$

which implies (9.9). 


\subsection{Proof of Theorem 9.1}

By definition $K \phi$ and $P \phi$ are currents in $\mathcal{W}^{0, *}\left(X^{\prime}\right)$ such that (9.6) and (9.7) hold for $\mu$ in $\mathcal{H o m}\left(\mathscr{O}_{\Omega^{\prime}} / \mathcal{J}, \mathcal{C} \mathcal{H}_{\Omega^{\prime}}^{Z^{\prime}}\right)$. We claim that

$$
K \phi \wedge R \wedge d z=\pi_{*}\left((B \wedge g \wedge H R(\zeta))_{N} \wedge \phi \wedge R(z) \wedge d z\right)
$$

and

$$
P \phi \wedge R \wedge d z=\pi_{*}\left((g \wedge H R(\zeta))_{N} \wedge \phi \wedge R(z) \wedge d z\right)
$$

here the left hand sides are defined in view of Remark 7.9, whereas the right hand sides have meaning by Lemma 9.2 and the fact that $R(z) \wedge d z$ is in $\mathcal{H o m}\left(\mathscr{O}_{\Omega^{\prime}} / \mathcal{J}, \mathcal{W}_{\Omega^{\prime}}^{Z^{\prime}}\right)$ by Corollary 6.3.

Recall from Lemma 6.2 that $R \wedge d z=b \mu$, where $\mu$ is a tuple of currents in $\mathcal{H o m}\left(\mathscr{O}_{\Omega^{\prime}} / \mathcal{J}, \mathcal{C H}_{\Omega^{\prime}}^{Z^{\prime}}\right)$ and $b$ is an almost semi-meromorphic matrix that is smooth generically on $Z^{\prime}$. Therefore (9.12) and (9.13) hold where $b$ is smooth, in view of Lemma 7.7, and since both sides are in $\mathcal{H o m}\left(\mathscr{O}_{\Omega^{\prime}} / \mathcal{J}, \mathcal{W}_{\Omega^{\prime}}^{Z^{\prime}}\right)$, the equalities hold everywhere by the SEP.

As in [6] we let $R^{\lambda}=\bar{\partial}|f|^{2 \lambda} \wedge U$ for $\operatorname{Re} \lambda \gg 0$. It has an analytic continuation to $\lambda=0$ and $R=\left.R^{\lambda}\right|_{\lambda=0}$. Notice that $R(z) \wedge B$ is well-defined since it is a tensor product with respect to the coordinates $z, \eta=\zeta-z$. Also $R(z) \wedge R^{\lambda}(\zeta) \wedge B$ admits such an analytic continuation and defines a pseudomeromorphic current ${ }^{4}$ when $\lambda=0$. Let $B_{k, k-1}$ be the component of $B$ of bidegree $(k, k-1)$.

Lemma 9.5 For all $k$,

$$
\left.B_{k, k-1} \wedge H R^{\lambda}(\zeta) \wedge R(z)\right|_{\lambda=0}=B_{k, k-1} \wedge H R(\zeta) \wedge R(z)
$$

Proof of Lemma 9.5 Notice that the equality holds outside $\Delta$. Let $T$ be the left hand side of (9.14). In view of Proposition 2.1 it is therefore enough to check that $\mathbf{1}_{\Delta} T=0$. Fix $j, k$ and let

$$
T_{\ell}=\left.B_{k, k-1} \wedge H R_{j}^{\lambda}(\zeta) \wedge R_{\ell}(z)\right|_{\lambda=0}
$$

Clearly $T_{\ell}=0$ if $\ell<p$ so first assume that $\ell=p$. Since $H R_{j}$ has bidegree $(j, j)$ in $\zeta$, the current vanishes unless $j+k \leq N$. Thus the total antiholomorphic degree is $\leq N-n+N-1$. On the other hand, the current has support on $\Delta \cap Z \times Z \simeq Z \times\{p t\}$ which has codimension $N+N-n$. Thus it vanishes by the dimension principle.

We now prove by induction over $\ell \geq p$ that $\mathbf{1}_{\Delta} T_{\ell}=0$. Note that by (6.6), outside of $Z_{\ell}, R_{\ell}(z)=\alpha_{\ell}(z) R_{\ell-1}(z)$, where $\alpha_{\ell}(z)$ is smooth. Thus, outside of $Z_{\ell} \times \Omega, T_{\ell}$ is a smooth form times $T_{\ell-1}$, and thus, by induction and (2.3), $\mathbf{1}_{\Delta} T_{\ell}$ has its support in $\Delta \cap\left(Z_{\ell} \times Z\right) \simeq Z_{\ell} \times\{p t\}$, which has codimension $\geq N+\ell+1$, see (6.3). On the other hand, the total antiholomorphic degree is $\leq \ell+j+k-1 \leq \ell+N-1$, so the current vanishes by the dimension principle. We conclude that (9.14) holds.

\footnotetext{
4 One can consider this current as $R(z) \wedge B$ multiplied by the residue of the almost semi-meromorphic current $U$ in (6.5), cf., [10, Section 4.4].
} 
By the same argument ${ }^{5}$ as for $[6,(5.2)]$ we have the equality

$\nabla_{f(z)}\left(\left(B \wedge g \wedge H R^{\lambda}(\zeta)\right)_{N} \wedge R(z) \wedge d z\right)=[\Delta]^{\prime} \wedge R(z) \wedge d z-\left(g \wedge H R^{\lambda}\right)_{N} \wedge R(z) \wedge d z$

also for our $R$, where $[\Delta]^{\prime}$ denotes the part of $[\Delta]$ where $d \eta_{i}=d \zeta_{i}-d z_{i}$ has been replaced ${ }^{6}$ by $d \zeta_{i}$. In view of (9.14) we can put $\lambda=0$ in (9.15), and then we get

$\nabla_{f(z)}\left((B \wedge g \wedge H R(\zeta))_{N} \wedge R(z) \wedge d z\right)=[\Delta]^{\prime} \wedge R(z) \wedge d z-(H R(\zeta) \wedge g)_{N} \wedge R(z) \wedge d z$

Multiplying (9.16) by the smooth form $\phi$, and using (9.12) and (9.13), we get

$$
\phi \wedge R \wedge d z=-\nabla_{f}(K \phi \wedge R \wedge d z)+K(\bar{\partial} \phi) \wedge R \wedge d z+P \phi \wedge R \wedge d z
$$

or equivalently,

$$
\phi \wedge \omega=-\nabla_{f}(K \phi \wedge \omega)+K(\bar{\partial} \phi) \wedge \omega+P \phi \wedge \omega .
$$

Multiplying by suitable holomorphic $\xi_{0}$ in $E_{p}^{*}$ such that $f_{p+1}^{*} \xi_{0}=0$, cf., Proposition 6.7, we see that $\phi \wedge h=\bar{\partial}(K \phi \wedge h)+K(\bar{\partial} \phi) \wedge h+P \phi \wedge h$ for all $h$ in $\omega_{X}$. Thus by definition (9.1) holds.

Since $\mathcal{W}_{X}^{0, *}$ is closed under multiplication by $\mathscr{O}_{X}$, we get that $\psi$ in $\mathcal{W}_{X}^{0, *}$ is in Dom $\bar{\partial}_{X}$ if and only if $-\nabla_{f}(\psi \wedge \omega)$ is in $\mathcal{W}_{X}^{n, *}$. Thus, we conclude from (9.17) that $K \phi$ is in Dom $\bar{\partial}_{X}$ since all the other terms but $-\nabla_{f}(K \phi \wedge \omega)$ are in $\mathcal{W}_{X}^{0, *}$.

\subsection{Intrinsic interpretation of $K$ and $P$}

So far we have defined $K$ and $P$ by means of currents in ambient space. We used this approach in order to avoid introducing push-forwards on a non-reduced space. However, we will sketch here how this can be done. We must first define the product space $X \times X^{\prime}$. Given a local embedding $i: X \rightarrow \Omega$ as before, we have an embedding $(i \times i): X \times X^{\prime} \rightarrow \Omega \times \Omega^{\prime}$ such that the structure sheaf is $\mathscr{O}_{\Omega \times \Omega^{\prime}} /\left(\mathcal{J}_{X}+\mathcal{J}_{X^{\prime}}\right)$. One can check that this sheaf is independent of the chosen embedding, i.e., $\mathscr{O}_{X \times X^{\prime}}$ is intrinsically defined. Thus we also have definitions of all the various sheaves on $X \times X^{\prime}$ like $\mathscr{E}_{X \times X^{\prime}}^{0, *}$. The projection $p: X \times X^{\prime} \rightarrow X^{\prime}$ is determined by $p^{*} \phi: \mathscr{O}_{X^{\prime}} \rightarrow$ $\mathscr{O}_{X \times X^{\prime}}$, which in turn is defined so that $p^{*} i^{*} \Phi=(i \times i)^{*} \pi^{*} \Phi$ for $\Phi$ in $\mathscr{O}_{\Omega^{\prime}}$, where $\pi: \Omega \times \Omega^{\prime} \rightarrow \Omega^{\prime}$ as before. Again one can check that this definition is independent of the embedding, and also extends to smooth $(0, *)$-forms $\phi$. Therefore, we have the well-defined mapping $p_{*}: \mathcal{C}_{X \times X^{\prime}}^{2 n, *+n} \rightarrow \mathcal{C}_{X^{\prime}}^{n, *}$, and clearly

$$
i_{*} p_{*}=\pi_{*}(i \times i)_{*}
$$

\footnotetext{
5 There is a sign error in $[6,(5.2)]$ due to $R(z) \wedge d z$ being odd with respect to the super structure. Since we here move $R(z) \wedge d z$ to the right, we get the correct sign.

6 This change is due to the fact that we do the same change of the differentials in the definition of $H$ and $B$ above.
} 
As before we have the isomorphism

$$
(i \times i)_{*}: \mathcal{W}_{X \times X^{\prime}}^{2 n, *} \simeq \mathcal{H o m}\left(\mathscr{O}_{\Omega \times \Omega^{\prime}} /\left(\mathcal{J}_{X}+\mathcal{J}_{X^{\prime}}\right), \mathcal{W}_{\Omega \times \Omega^{\prime}}^{Z \times Z^{\prime}}\right)
$$

As in the proof of Lemma 9.2 we see that $\pi_{*}$ maps a current in $\mathcal{W}_{\Omega \times \Omega^{\prime}}^{Z \times Z^{\prime}}$ annihilated by $\mathcal{J}_{X^{\prime}}$ to a current in $\mathcal{H o m}\left(\mathscr{O}_{\Omega} / \mathcal{J}, \mathcal{W}_{\Omega^{\prime}}^{Z^{\prime}}\right)$. It follows by (9.18) that

$$
p_{*}: \mathcal{W}_{X \times X^{\prime}}^{2 n, *+n} \rightarrow \mathcal{W}_{X^{\prime}}^{n, *}
$$

Now, take $h$ in $\omega_{X^{\prime}}^{n}$ and let $\mu=i_{*} h$. Then, cf., the proof of Lemma 9.2,

$(B \wedge g \wedge H R(\zeta))_{N} \wedge \phi(\zeta) \wedge \mu(z)=(i \times i)_{*}(\vartheta(B \wedge g \wedge H) \wedge \omega(\zeta) \wedge \phi(\zeta) \wedge h)$

Thus we can define $K \phi$ intrinsically by

$$
K \phi \wedge h=p_{*}(\vartheta(B \wedge g \wedge H) \wedge \omega(\zeta) \wedge \phi(\zeta) \wedge h(z))
$$

From above it follows that $K \phi \wedge h$ is in $\mathcal{W}_{X^{\prime}}^{n, *}$. In the same way we can define $P \phi$ by

$$
P \phi \wedge h=p_{*}(\vartheta(g \wedge H) \wedge \omega(\zeta) \wedge \phi(\zeta) \wedge h(z))
$$

It is natural to write

$$
K \phi(z)=\int_{\zeta} \vartheta(B \wedge g \wedge H) \wedge \omega(\zeta) \wedge \phi(\zeta), \quad P \phi(z)=\int_{\zeta} \vartheta(g \wedge H) \wedge \omega(\zeta) \wedge \phi(\zeta)
$$

although the formal meaning is given by (9.19) and (9.20).

\section{Regularity of solutions on $X_{\text {reg }}$}

We have already seen, cf., Proposition 9.3 , that $P \phi$ is always a smooth form. We shall now prove that $K$ preserves regularity on $X_{\text {reg }}$. More precisely,

Theorem 10.1 If $\phi$ in $\mathcal{W}_{X}^{0, *}$ is smooth near a point $x \in X_{\text {reg, }}^{\prime}$, then $K \phi$ in Theorem 9.1 is smooth near $x$.

Throughout this section, let us choose local coordinates $(\zeta, \tau)$ and $(z, w)$ at $x$ corresponding to the variables $\zeta$ and $z$ in the integral formulas, so that $Z=\{(\zeta, \tau) ; \tau=$ $0\}$.

Lemma 10.2 Let $B^{\epsilon}:=\chi\left(|\zeta-z|^{2} / \epsilon\right) B$, and assume that $\phi$ has compact support in our coordinate neighborhood. Then $K \phi$ can be approximated by the smooth forms

$$
K^{\epsilon} \phi:=\pi_{*}\left(\left(B^{\epsilon} \wedge g \wedge H R\right)_{N} \wedge \phi\right) .
$$


Notice that here we cut away the diagonal $\Delta^{\prime}$ in $Z \times Z^{\prime}$ times $\mathbb{C}_{\tau} \times \mathbb{C}_{w}$ in contrast to Proposition 9.4, where we only cut away the diagonal $\Delta$ in $\Omega \times \Omega^{\prime}$.

Proof Clearly $B^{\epsilon}$ is smooth so that each $K^{\epsilon} \phi$ is smooth in a full neighborhood in $\Omega^{\prime}$ of $x$. Let $T=\mu(z, w) \wedge(H R(\zeta, \tau) \wedge B \wedge g)_{N} \wedge \phi$, and let $W=\Delta^{\prime} \times \mathbb{C}_{\tau} \times \mathbb{C}_{w}$. Since $\mu(z, w) \otimes R(\zeta, \tau)$ has support on $\{w=\tau=0\}, T=\mathbf{1}_{\{w=\tau=0\}} T$. Therefore, $\mathbf{1}_{W} T=\mathbf{1}_{W} \mathbf{1}_{\{w=\tau=0\}} T=0$ since $W \cap\{w=\tau=0\} \subset \Delta$ and $\mathbf{1}_{\Delta} T=0$ by definition, cf., Proposition 2.1 (i). Now notice that $\mathbf{1}_{W} T=0$ implies (9.11) and in turn (9.9) with our present choice of $B^{\epsilon}$.

We first consider a simple but nontrivial example of Theorem 10.1.

Example 10.3 Let $X=\mathbb{C}_{\zeta} \subset \mathbb{C}_{\zeta, \tau}^{2}$ and $\mathcal{J}=\left(\tau^{m+1}\right)$. Then $R=\bar{\partial}\left(1 / \tau^{m+1}\right)$. For an arbitrary point $(z, w)$ we can choose the Hefer form

$$
H=\frac{1}{2 \pi i} \sum_{j=0}^{m} \tau^{m-k} w^{k} d \tau
$$

From the Bochner-Martinelli form $B$ we only get a contribution from the term

$$
B_{1}=\frac{1}{2 \pi i} \frac{(\bar{\zeta}-\bar{z}) d \zeta+(\bar{\tau}-\bar{w}) d \tau}{|\zeta-z|^{2}+|\tau-w|^{2}}
$$

Let $\Omega^{\prime} \subset \subset \Omega$ be open balls with center at the origin, and let $\varphi=\varphi\left(|\zeta|^{2}+|\tau|^{2}\right)$ be a smooth cutoff function with support in $\Omega$ that is $\equiv 1$ in a neighborhood of $\overline{\Omega^{\prime}}$. Then we can choose a holomorphic weight $g=\varphi+\cdots$, see, [6, Example 5.1] with respect to $\Omega^{\prime}$, and with support in $\Omega$. Now $1, \tau, \ldots, \tau^{m}$ is a set of generators for $\mathscr{O}_{X}$ over $\mathscr{O}_{Z}$. Assume that

$$
\phi=\left(\hat{\phi}_{0}(\zeta) \otimes 1+\cdots+\hat{\phi}_{m}(\zeta) \otimes \tau^{m}\right) d \bar{\zeta}
$$

is a smooth $(0,1)$-form. We want to compute $K \phi$. We know that

$$
K \phi=a_{0}(z) \otimes 1+\cdots+a_{m}(z) \otimes w^{m}
$$

with $a_{k}(z)$ in $\mathcal{W}_{Z}^{0,0}$. By Lemma 10.2 and its proof, we have smooth $K^{\epsilon} \phi(z, w)$ in $\Omega^{\prime}$ such that

$$
K^{\epsilon} \phi \wedge d z \wedge d w \wedge \bar{\partial} \frac{1}{w^{m+1}} \rightarrow K \phi \wedge d z \wedge d w \wedge \bar{\partial} \frac{1}{w^{m+1}} .
$$

It follows that

$$
a_{k}(z)=\left.\lim _{\epsilon \rightarrow 0} \frac{1}{k !} \frac{\partial^{k}}{\partial w^{k}} K^{\epsilon} \phi(z, w)\right|_{w=0} .
$$


Notice that

$$
\begin{aligned}
(B \wedge g \wedge H R(\tau))_{2} & =B_{1} \wedge g_{0,0} \wedge H \wedge \bar{\partial} \frac{1}{\tau^{m+1}} \\
& =-\varphi \bar{\partial} \frac{1}{\tau^{m+1}} \wedge \frac{1}{(2 \pi i)^{2}} \sum_{\ell=0}^{m} \tau^{m-\ell} w^{\ell} d \tau \wedge \frac{(\bar{\zeta}-\bar{z}) d \zeta+(\bar{\tau}-\bar{w}) d \tau}{|\zeta-z|^{2}+|\tau-w|^{2}} \\
& =-\varphi \bar{\partial} \frac{d \tau}{\tau^{m+1}} \wedge \frac{1}{(2 \pi i)^{2}} \sum_{\ell=0}^{m} \tau^{m-\ell} w^{\ell} \wedge \frac{(\bar{\zeta}-\bar{z}) d \zeta}{|\zeta-z|^{2}+|\tau-w|^{2}} .
\end{aligned}
$$

For each fixed $\epsilon>0,|\zeta-z|>0$ on supp $\chi_{\epsilon}$, cf., Lemma 10.2, so we have

$$
\begin{aligned}
& K^{\epsilon} \phi(z, w) \\
& =\int_{\zeta, \tau} \varphi \frac{1}{(2 \pi i)^{2}} \sum_{\ell=0}^{m} \bar{\partial} \frac{d \tau}{\tau^{\ell+1}} \wedge w^{\ell} \chi \epsilon \frac{(\bar{\zeta}-\bar{z}) d \bar{\zeta} \wedge d \zeta}{|\zeta-z|^{2}+|\tau-w|^{2}} \wedge \sum_{k=0}^{m} \hat{\phi}_{k}(\zeta) \otimes \tau^{k} .
\end{aligned}
$$

A simple computation yields that

$$
K^{\epsilon} \phi(z, w)=\sum_{k=0}^{m} a_{k}^{\epsilon}(z) \otimes w^{k}+\mathscr{O}(\bar{w}),
$$

where

$$
a_{k}^{\epsilon}(z)=\frac{1}{2 \pi i} \int_{\zeta} \varphi\left(|\zeta|^{2}\right) \chi_{\epsilon} \frac{\hat{\phi}_{k}(\zeta) d \bar{\zeta} \wedge d \zeta}{\zeta-z}
$$

Letting $\epsilon$ tend to 0 we get $K \phi$ as in (10.1), where

$$
a_{k}(z)=\frac{1}{2 \pi i} \int_{\zeta} \varphi\left(|\zeta|^{2}\right) \frac{\hat{\phi}_{k}(\zeta) d \bar{\zeta} \wedge d \zeta}{\zeta-z} .
$$

It is well-known that these Cauchy integrals $a_{k}(z)$ are smooth solutions to $\bar{\partial} v=\hat{\phi}_{k} d \bar{z}$ in $Z^{\prime}=Z \cap \Omega^{\prime}$. Thus $K \phi$ is smooth.

Remark 10.4 The terms $\mathscr{O}(\bar{w})$ in the expansion (10.4) of $K^{\epsilon} \phi(z, w)$ do not converge to smooth functions in general when $\epsilon \rightarrow 0$. For a simple example, take $\phi=\zeta d \bar{\zeta} \otimes \tau^{m}$. Then $K^{\epsilon} \phi(0, w)$ tends to

$$
w^{m} \int \varphi\left(|\zeta|^{2}\right) \frac{1}{2 \pi i} \frac{|\zeta|^{2} d \bar{\zeta} \wedge d \zeta}{|\zeta|^{2}+|w|^{2}}
$$

which is a smooth function of $w$ plus (a constant times) $w^{m}|w|^{2} \log |w|^{2}$, and thus not smooth. However, it is certainly in $C^{m}$. One can check that $K \phi(z, w)=$ 
$\lim _{\epsilon \rightarrow 0^{+}} K^{\epsilon} \phi(z, w)$ exists pointwise and defines a function in at least $C^{m}$ and that our solution can be computed from this limit. In fact, by a more precise computation we get from (10.3) that

$$
K^{\epsilon} \phi(z, w)=\sum_{k=0}^{m} \int_{\zeta} \varphi\left(|\zeta|^{2}\right) \chi_{\epsilon} \frac{1}{2 \pi i} \frac{(\bar{\zeta}-\bar{z}) \hat{\phi}_{k}(\zeta) d \bar{\zeta} \wedge d \zeta}{|\zeta-z|^{2}+|w|^{2}} w^{k} \sum_{j=0}^{m-k}\left(\frac{|w|^{2}}{|\zeta-z|^{2}+|w|^{2}}\right)^{j}
$$

It is now clear that we can let $\epsilon \rightarrow 0$. By a simple computation we then get

$$
\begin{aligned}
K \phi(z, w)= & \sum_{k=0}^{m} C \hat{\phi}_{k}(z) \otimes w^{k} \\
& -\sum_{k=0}^{m} \int_{\zeta} \varphi\left(|\zeta|^{2}\right) \frac{1}{2 \pi i} \frac{\hat{\phi}_{k}(\zeta) d \bar{\zeta} \wedge d \zeta}{\zeta-z} w^{k}\left(\frac{|w|^{2}}{|\zeta-z|^{2}+|w|^{2}}\right)^{m-k+1} .
\end{aligned}
$$

Let $\psi=\varphi \hat{\phi}_{k}$. Then the $k$ th term in the second sum is equal to

$$
b(z, w)=\frac{1}{2 \pi i} \int_{\zeta} \frac{\psi(z+\zeta) d \bar{\zeta} \wedge d \zeta}{\zeta} w^{k}\left(\frac{|w|^{2}}{|\zeta|^{2}+|w|^{2}}\right)^{m-k+1} .
$$

If we integrate outside the unit disk, then we certainly get a smooth function. Thus it is enough to consider the integral over the disk. Moreover, if $\psi(z+\zeta)=\mathscr{O}\left(|\zeta|^{M}\right)$ for a large $M$, then the integral is at least $C^{m}$. By a Taylor expansion of $\psi(z+\zeta)$ at the point $z$, we are thus reduced to consider

$$
\int_{|\zeta|<1} \frac{\zeta^{\alpha} \bar{\zeta}^{\beta}}{\zeta}\left(\frac{|w|^{2}}{|\zeta|^{2}+|w|^{2}}\right)^{m-k+1}
$$

For symmetry reasons, they vanish, except when $\alpha=\beta+1$. Thus we are left with

$$
\int_{|\zeta|<1}|\zeta|^{2 \beta}\left(\frac{|w|^{2}}{|\zeta|^{2}+|w|^{2}}\right)^{m-k+1} w^{k}=C w^{k}|w|^{2(m-k+1)} \int_{0}^{1} \frac{s^{\beta} d s}{\left(s+|w|^{2}\right)^{m-k+1}}
$$

for non-negative integers $\beta$. The right hand side is a smooth function of $w$ if $\beta \leq$ $m-k-1$ and a smooth function plus

$$
C w^{k}|w|^{2(\beta+1)} \log |w|^{2}
$$

if $\beta \geq m-k$. The worst case therefore is when $k=m$ and $\beta=0$; then we have $w^{m}|w|^{2} \log |w|^{2}$ that we encountered above.

Proposition 10.5 Let $z, w$ be coordinates at a point $x \in X_{\text {reg }}$ such that $Z=\{w=0\}$ and $x=(0,0)$. If $\phi$ is smooth, and has support where the local coordinates are defined, then 


$$
v^{\epsilon}(z, w)=\int_{\zeta} \chi\left(|\zeta-z|^{2} / \epsilon\right)(H R \wedge B \wedge g)_{N} \wedge \phi,
$$

is smooth for $\epsilon>0$, and for each multiindex $\ell$ there is a smooth form $v_{\ell}$ such that

$$
\left.\partial_{w}^{\ell} v^{\epsilon}\right|_{w=0} \rightarrow v_{\ell}
$$

as currents on $Z$.

Taking this proposition for granted we can conclude the proof of Theorem 10.1.

Proof of Theorem 10.1 If $\phi \equiv 0$ in a neighborhood of $x \in X_{\text {reg }}^{\prime}$, then $K \phi$ is smooth near $x$, cf., the proof of Proposition 9.4. Thus, it is sufficient to prove Theorem 10.1 assuming that $\phi$ is smooth and has support near $x$.

Recall that given a minimal generating set $1, w^{\alpha_{1}}, \ldots, w^{\alpha_{\nu-1}}$, one gets the coefficients $\hat{v}_{j}^{\epsilon}$ in the representation

$$
v^{\epsilon}=\hat{v}_{0}^{\epsilon} \otimes 1+\cdots+\hat{v}_{\nu-1}^{\epsilon} \otimes w^{\alpha_{\nu-1}}
$$

from $\left.\partial_{w}^{\ell} v^{\epsilon}\right|_{w=0},|\ell| \leq M$ by a holomorphic matrix, cf., the proof of Lemma 4.7. It thus follows from Proposition 10.5 that there are smooth $\hat{v}_{j}$ such that $\hat{v}_{j}^{\epsilon} \rightarrow \hat{v}_{j}$ as currents on $Z$. Let $v=\hat{v}_{0} \otimes 1+\cdots+\hat{v}_{v-1} \otimes w^{\alpha_{\nu-1}}$. In view of (2.14), $v^{\epsilon} \wedge \mu \rightarrow v \wedge \mu$ for all $\mu$ in $\mathcal{H o m}\left(\mathscr{O}_{\Omega} / \mathcal{J}, \mathcal{C} \mathcal{H}_{\Omega}^{Z}\right)$. From Lemma 10.2 we conclude that $v \wedge \mu=K \phi \wedge \mu$ for all such $\mu$. Thus $K \phi=v$ in $\mathcal{W}_{X}^{0, *}$ and hence $K \phi$ is smooth.

Proof of Proposition 10.5 Assume that $X$ is embedded in $\Omega \subset \mathbb{C}_{\zeta^{\prime}, \tau^{\prime}}^{N}$. After a suitable rotation we can assume that $Z$ is the graph $\tau^{\prime}=\psi\left(\zeta^{\prime}\right)$. The Bochner-Martinelli kernel in $\Omega$ is rotation invariant, so it is

$$
B=\sigma+\sigma \wedge \bar{\partial} \sigma+\sigma \wedge(\bar{\partial} \sigma)^{2}+\cdots,
$$

where

$$
\sigma=\frac{\left(\bar{\zeta}^{\prime}-\bar{z}^{\prime}\right) \cdot d \zeta^{\prime}+\left(\bar{\tau}^{\prime}-\bar{w}^{\prime}\right) \cdot d \tau^{\prime}}{\left|\zeta^{\prime}-z^{\prime}\right|^{2}+\left|\tau^{\prime}-w^{\prime}\right|^{2}}
$$

We now choose the new coordinates $\zeta=\zeta^{\prime}, \tau=\tau^{\prime}-\psi\left(\zeta^{\prime}\right)$ in $\Omega$, so that $Z=$ $\{(\zeta, \tau) ; \tau=0\}$.

Recall that on $X_{\text {reg }}$ we have that $R \wedge d z$ is a smooth form times $\mu=\left(\mu_{1}, \ldots, \mu_{m}\right)$, where $\mu_{j}$ is a generating set for $\mathcal{H} \operatorname{om}\left(\mathscr{O}_{\Omega} / \mathcal{J}, \mathcal{C} \mathcal{H}_{\Omega}^{Z}\right)$. Thus we are to compute $\left.\partial_{w}^{\ell}\right|_{w=0}$ of integrals like

$$
\int_{\zeta, \tau} \bar{\partial} \frac{d \tau}{\tau^{\alpha+1}} \wedge B_{k}^{\epsilon} \wedge \phi(\zeta, z, w, \tau),
$$

where $k \leq n$ and $\phi$ is smooth with compact support near $x$. It is clear that the symbols $\bar{\tau}, \bar{w}, d \bar{\tau}$ can be omitted in the expression for

$$
B^{\epsilon}=\chi_{\epsilon} B=\chi\left(|\zeta-z|^{2} / \epsilon\right) B
$$


since $\bar{\tau}$ and $d \bar{\tau}$ annihilate $\bar{\partial}\left(1 / \tau^{\alpha+1}\right)$, and since we only take holomorphic derivatives with respect to $w$ and set $w=0$.

Let us write $\psi(\zeta)-\psi(z)=A(\zeta, z) \eta$, where $\eta:=\zeta-z$ is considered as a column matrix and $A$ is a holomorphic $(N-n) \times n$-matrix. Then

$$
\sigma=\frac{\eta^{*} v}{|\zeta-z|^{2}+|\tau-w+\psi(\zeta)-\psi(z)|^{2}}
$$

where $v$ is the $(1,0)$-form valued column matrix

$$
v=d \zeta+A^{*} d(\tau+\psi(\zeta))
$$

Since $\eta^{*} v$ is a $(1,0)$-form we have that

$$
B_{k}^{\epsilon}=\chi \epsilon \frac{\eta^{*} v \wedge\left(\left(d \eta^{*}\right) v+\eta^{*} \bar{\partial} v\right)^{k-1}}{\left(|\zeta-z|^{2}+|\tau-w+\psi(\zeta)-\psi(z)|^{2}\right)^{k}}
$$

\section{Lemma 10.6 Let}

$$
\xi^{i}=\xi_{1}^{i} \frac{\partial}{\partial \zeta_{1}}+\cdots+\xi_{n}^{i} \frac{\partial}{\partial \zeta_{n}}
$$

be smooth $(1,0)$-vector fields, and let $L_{i}=L_{\xi^{i}}$ be the associated Lie derivatives for $i=1, \ldots, \rho$. Let

$$
\gamma_{k}:=\eta^{*} v \wedge\left(\left(d \eta^{*}\right) v+\eta^{*} \bar{\partial} v\right)^{k-1}
$$

If we have a modification $\pi: \tilde{W} \rightarrow \Omega \times \Omega$ such that locally $\pi^{*} \eta=\eta_{0} \eta^{\prime}$, where $\eta_{0}$ is a holomorphic function, then

$$
\pi^{*}\left(L_{1} \cdots L_{\rho} \gamma_{k}\right)=\bar{\eta}_{0}^{k} \beta
$$

where $\beta$ is smooth.

Recall that if $a$ is a form, then $L_{\xi} a=d(\xi \neg a)+\xi \neg(d a)$, and that $L_{\xi}(\beta \neg a)=$ $[\xi, \beta] \neg a+\beta \neg\left(L_{\xi} a\right)$ if $\beta$ is another vector field.

Proof Introduce a nonsense basis $e$ and its dual $e^{*}$ and consider the exterior algebra spanned by $e_{j}, e_{\ell}^{*}$, and the cotangent bundle. Let

$$
c_{\ell}=\eta^{*} e \wedge\left(\left(d \eta^{*}\right) e\right)^{\ell-1} .
$$

Notice that $\gamma_{k}$ is a sum of terms like

$$
\left(v e^{*} \neg\right)^{\ell} c_{\ell} \wedge\left(\eta^{*} \bar{\partial} v\right)^{k-\ell} .
$$


Since $L_{i} c_{\ell}=0$ and $L_{i}\left(\eta^{*} b\right)=\eta^{*} L_{i} b$ it follows after a finite number of applications of $L_{i}$ 's that we get

$$
\left(v_{1} e^{*}\right) \neg \cdots\left(v_{\ell} e^{*}\right) \neg c_{\ell}\left(\eta^{*} b_{1}\right) \cdots\left(\eta^{*} b_{k-\ell}\right),
$$

where $v_{j}$ and $b_{j}$ are smooth. Since

$$
\pi^{*} c_{\ell}=\bar{\eta}_{0}^{\ell}\left(\eta^{\prime}\right)^{*} e \wedge\left(d\left(\eta^{\prime}\right)^{*} e\right)^{\ell-1}
$$

the lemma now follows.

We note that $\eta^{*}\left(I+A^{*} A\right) \eta=|\zeta-z|^{2}+|\psi(\zeta)-\psi(z)|^{2}$. Thus, differentiating (10.5) with respect to $w$, setting $w=0$, and evaluating the residue with respect to $\tau$ using (2.10), we obtain a sum of integrals like

$$
\int_{\zeta} \chi_{\epsilon} \frac{\left(\eta^{*} a_{1}\right) \cdots\left(\eta^{*} a_{t+1}\right) \wedge \gamma_{k} \wedge \phi}{\left(\eta^{*}\left(I+A^{*} A\right) \eta\right)^{k+t+1}}
$$

where $a_{1}, \ldots, a_{t+1}$ are column vectors of smooth functions. We must prove that the limit of such integrals when $\epsilon \rightarrow 0$ are smooth in $z$.

Lemma 10.7 Let

$$
I_{\ell}^{r, s}=\int \chi_{\epsilon} \frac{\left(\eta^{*} a_{1}\right) \cdots\left(\eta^{*} a_{r}\right) \mathscr{O}\left(|\eta|^{2 s}\right) \tilde{\gamma}_{k} \wedge \phi}{\Phi^{k+\ell}},
$$

where $a_{1}, \ldots, a_{r}$ are tuples of smooth functions, $\tilde{\gamma}_{k}=L_{1} \cdots L_{\rho} \gamma_{k}$, where $L_{i}=L_{\xi_{i}}$ are Lie derivatives with respect to smooth $(1,0)$-vector fields $\xi^{i}$ as above for $i=$ $1, \ldots, \rho, \phi$ is a test form with support close to $z$, and $\Phi:=\eta^{*}\left(I+A^{*} A\right) \eta$. If $r \geq 1$ and $r+s \geq \ell+1$, then we have the relation

$$
I_{\ell+1}^{r, s}=I_{\ell}^{r-1, s}+I_{\ell+1}^{r-1, s+1}+I_{\ell}^{r, s-1}+o(1)
$$

when $\epsilon \rightarrow 0$.

Proof If

$$
\xi=a_{r}^{t}\left(I+A^{*} A\right)^{-t} \frac{\partial}{\partial \zeta}
$$

and $L=L_{\xi}$, then using that $\Phi=\eta^{t}\left(I+A^{*} A\right)^{t} \bar{\eta}$, one obtains that

$$
L \Phi=\eta^{*} a_{r}+\mathscr{O}\left(|\eta|^{2}\right)
$$

Thus

$$
I_{\ell+1}^{r, s}=\int \chi_{\epsilon}\left(\eta^{*} a_{1}\right) \cdots\left(\eta^{*} a_{r-1}\right) \mathscr{O}\left(|\eta|^{2 s}\right) \tilde{\gamma}_{k} \wedge \phi L \frac{1}{\Phi^{k+\ell}}+I_{\ell+1}^{r-1, s+1}
$$


in view of (10.7). We now integrate by parts by $L$ in the integral. If a derivative with respect to $\zeta_{j}$ falls on some $\eta^{*} a_{i}$, we get a term $I_{\ell}^{r-1, s}$. If it falls on $\mathscr{O}\left(|\eta|^{2 s}\right)$ we get either $\mathscr{O}\left(|\eta|^{2(s-1)}\right)$ times $\eta^{*} b$, for some tuple $b$ of smooth functions, and this gives rise to the term $I_{\ell}^{r, s-1}$ or $\mathscr{O}\left(|\eta|^{2 s}\right)$, and this gives rise to another term $I_{\ell}^{r-1, s}$. If it falls on $\phi$ or $\tilde{\gamma}_{k}$ we get an additional term $I_{\ell}^{r-1, s}$. The only possibility left is when the derivative falls on $\chi_{\epsilon}=\chi\left(|\eta|^{2} / \epsilon\right)$. It remains to show that an integral of the form

$$
\int_{\zeta, z} \chi^{\prime}\left(|\eta|^{2} / \epsilon\right) \frac{\left(\eta^{*} a_{1}\right) \cdots\left(\eta^{*} a_{r-1}\right)\left(\eta^{*} b\right)}{\epsilon} \frac{\mathscr{O}\left(|\eta|^{2 s}\right) \gamma_{k} \wedge \phi}{\Phi^{k+\ell}}
$$

tends to 0 , where the factor $\eta^{*} b$ comes from the derivative of $|\eta|^{2}$. We now choose a resolution $\widetilde{V} \rightarrow \Omega \times \Omega$ such that $\eta=\eta_{0} \eta^{\prime}$ where $\eta^{\prime}$ is non-vanishing and $\eta_{0}$ is (locally) a monomial. Notice that $\pi^{*} \Phi=\left|\eta_{0}\right|^{2} \Phi^{\prime}$ where $\Phi^{\prime}$ is smooth and strictly positive. In view of Lemma 10.6 we thus obtain integrals of the form

$$
\int_{\widetilde{V}} \chi^{\prime}\left(\left|\eta_{0}\right|^{2} v / \epsilon\right) \frac{1}{\epsilon} \frac{\bar{\eta}_{0}^{r+s-\ell}}{\eta_{0}^{k+\ell-s}} \alpha
$$

where $v$ is smooth and strictly positive and $\alpha$ is smooth.

In order to see that the limit of (10.8) tends to 0 , we note first that if we let

$$
\tilde{\chi}(s)=s \chi^{\prime}(s)+\chi(s),
$$

then just as $\chi, \tilde{\chi}$ is also a smooth function on $[0, \infty)$ that is 0 in a neighborhood of 0 and 1 in a neighborhood of $\infty$. By assumption, $r+s-\ell-1 \geq 0$. Since the principal value current $1 / f^{m}$ acting on a test form $\beta$ can be defined as

$$
\lim _{\epsilon \rightarrow 0^{+}} \int \chi\left(|f|^{2} v / \epsilon\right) \frac{\beta}{f^{m}}
$$

for any cut-off function as above, the principal value current $1 / \eta_{0}^{k+\ell-s}$ acting on $\bar{\eta}_{0}^{r+s-\ell-1} \alpha$ equals

$$
\lim _{\epsilon \rightarrow 0^{+}} \int_{\widetilde{V}} \chi\left(\left|\eta_{0}\right|^{2} v / \epsilon\right) \frac{\bar{\eta}_{0}^{r+s-\ell-1}}{\eta_{0}^{k+\ell-s}} \alpha=\lim _{\epsilon \rightarrow 0^{+}} \int_{\widetilde{V}} \tilde{\chi}\left(\left|\eta_{0}\right|^{2} v / \epsilon\right) \frac{\bar{\eta}_{0}^{r+s-\ell-1}}{\eta_{0}^{k+\ell-s}} \alpha .
$$

Taking the difference between the left and right hand side, we conclude that (10.8) tends to 0 when $\epsilon \rightarrow 0$.

Now we can conclude the proof of Proposition 10.5. From the beginning we have $I_{\ell}^{\ell, 0}$. After repeated applications of (10.6) we end up with

$$
I_{\ell}^{0, \ell}+I_{\ell-1}^{0, \ell-1}+\cdots+I_{0}^{0,0}+o(1)
$$


However, any of these integrals has an integrable kernel even when $\epsilon=0$. This means that we are back to the case in [6, Lemma 6.2], and so the limit integral is smooth in $z$.

\section{A fine resolution of $\mathscr{O}_{X}$}

We first consider a generalization of Theorem 9.1 .

Lemma 11.1 Assume that $\phi \in \mathcal{W}^{0, k}(X) \cap \mathscr{E}_{X}^{0, k}\left(X_{\text {reg }}\right) \cap$ Dom $\bar{\partial}_{X}$ and that $K \phi$ is in Dom $\bar{\partial}_{X}$ (or just in Dom $\bar{\partial}$ ). Then (9.1) holds on $X^{\prime}$.

Proof Let $\chi_{\delta}$ be functions as before that cut away $X_{\text {sing }}$. From Koppelman's formula (9.1) for smooth forms we have

$\chi_{\delta} \phi \wedge h=\bar{\partial}\left(K\left(\chi_{\delta} \phi\right)\right) \wedge h+K\left(\chi_{\delta} \bar{\partial} \phi\right) \wedge h+P\left(\chi_{\delta} \phi\right) \wedge h+K\left(\bar{\partial} \chi_{\delta} \wedge \phi\right) \wedge h, h \in \omega_{X}^{n}$,

for $z \in X_{\text {reg }}^{\prime}$. Clearly the left hand side tends to $\phi \wedge h$ when $\delta \rightarrow 0$. From Lemma 9.2 it follows that $K\left(\chi_{\delta} \phi\right) \wedge h \rightarrow K \phi \wedge h$. Thus the first term on the right hand side of (11.1) tends to $\bar{\partial}(K \phi) \wedge h$. In the same way the second and third terms on the right hand side tend to $K(\bar{\partial} \phi) \wedge h$ and $P \phi \wedge h$, respectively. It remains to show that the last term tends to 0 . If $z$ belongs to a fixed compact subset of $X_{\text {reg }}^{\prime}$, then $B$ is smooth in (9.5) when $\zeta$ is in supp $\bar{\partial} \chi_{\delta}$ for small $\delta$. Hence it suffices to see that

$$
R(\zeta) \wedge d \zeta \wedge \bar{\partial} \chi_{\delta} \wedge \phi(\zeta) \wedge i_{*} h \rightarrow 0
$$

and since this is a tensor product of currents, it suffices to see that

$$
R(\zeta) \wedge d \zeta \wedge \bar{\partial} \chi_{\delta} \wedge \phi(\zeta) \rightarrow 0
$$

or equivalently, $\omega(\zeta) \wedge \bar{\partial} \chi_{\delta} \wedge \phi(\zeta) \rightarrow 0$, which follows by Lemma 8.4 since $\phi$ is in Dom $\bar{\partial}_{X}$. We have thus proved that

$$
\chi_{\delta} \phi \wedge h=\chi_{\delta} \bar{\partial}(K \phi) \wedge h+\chi_{\delta} K(\bar{\partial} \phi) \wedge h+\chi_{\delta} P \phi \wedge h .
$$

The first term on the right hand side is equal to $\bar{\partial}\left(\chi_{\delta} K \phi \wedge h\right)-\bar{\partial} \chi_{\delta} \wedge K \phi \wedge h$, where the latter term tends to 0 if $K \phi$ is in Dom $\bar{\partial}_{X}$ or just in Dom $\bar{\partial}$, cf., Lemma 8.4. Thus we get

$$
\phi \wedge h=\bar{\partial}(K \phi) \wedge h+K(\bar{\partial} \phi) \wedge h+P \phi \wedge h, \quad h \in \omega_{X}^{n},
$$

which precisely means that (9.1) holds.

Definition 11.2 We say that a $(0, q)$-current $\phi$ on an open set $\mathcal{U} \subset X$ is a section of $\mathscr{A}_{X}^{q}$ over $\mathcal{U}, \phi \in \mathscr{A}^{q}(\mathcal{U})$, if, for every $x \in \mathcal{U}$, the germ $\phi_{x}$ can be written as a finite sum of terms

$$
\xi_{v} \wedge K_{v}\left(\cdots \xi_{2} \wedge K_{2}\left(\xi_{1} \wedge K_{1}\left(\xi_{0}\right)\right)\right)
$$


where $\xi_{j}$ are smooth $(0, *)$-forms and $K_{j}$ are integral operators with kernels $k_{j}(\zeta, z)$ at $x$, defined as above, and such that $\xi_{j}$ has compact support in the set where $z \mapsto k_{j}(\zeta, z)$ is defined.

Clearly $\mathscr{A}_{X}^{*}$ is closed under multiplication by $\xi$ in $\mathscr{E}_{X}^{0, *}$. It follows from (9.8) that $\mathscr{A}_{X}^{*}$ is a subsheaf of $\mathcal{W}_{X}^{0, *}$ and from Theorem 10.1 that $\mathscr{A}_{X}^{k}=\mathscr{E}_{X}^{0, *}$ on $X_{\text {reg }}$. Clearly any operator $K$ as above maps $\mathscr{A}_{X}^{*+1} \rightarrow \mathscr{A}_{X}^{*}$.

Lemma 11.3 If $\phi$ is in $\mathscr{A}_{X}$, then $\phi$ and $K \phi$ are in Dom $\bar{\partial}_{X}$.

Proof Notice that [6, Lemma 6.4] holds in our case by verbatim the same proof, since we have access to the dimension principle for (tensor products of) pseudomeromorphic $(n, *)$-currents, and the computation rule (2.3), cf., the comment after Definition 5.7. Since $\mathscr{A}_{X}^{*}=\mathscr{E}_{X}^{0, *}$ on $X_{\text {reg }}$ it is enough by Lemma 8.4 to check that $\bar{\partial} \chi_{\delta} \wedge \omega \wedge \phi \rightarrow 0$, and this precisely follows from [6, Lemma 6.4].

In view of Lemmas 11.1 and 11.3 we have

Proposition 11.4 Let $K, P$ be integral operators as in Theorem 9.1. Then

$$
K: \mathscr{A}^{k+1}(X) \rightarrow \mathscr{A}^{k}\left(X^{\prime}\right), \quad P: \mathscr{A}^{k}(X) \rightarrow \mathscr{E}^{0, k}\left(X^{\prime}\right),
$$

and the Koppelman formula (9.1) holds.

Proof of Theorem 1.1 By definition, it is clear that $\mathscr{A}_{X}^{k}$ are modules over $\mathscr{E}_{X}^{0, k}$, and by

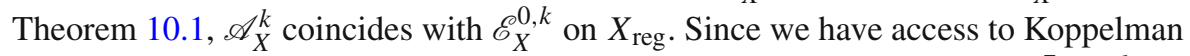
formulas, precisely as in the proof of [6, Theorem 1.2] we can verify that $\bar{\partial}: \mathscr{A}_{X}^{k} \rightarrow$ $\mathscr{A}_{X}^{k+1}$.

It remains to prove that (1.2) is exact. We choose locally a weight $g$ that is holomorphic in $z$, so the term $P \phi$ vanishes if $\phi$ is in $\mathscr{A}_{X}^{k}, k \geq 1$, and is holomorphic in $z$ when $k=0$. Assume that $\phi$ is in $\mathscr{A}_{X}^{k}$ and $\bar{\partial} \phi=0$. If $k \geq 1$, then $\bar{\partial} K \phi=\phi$, and if $k=0$, then $\phi=P \phi$.

\subsection{Global solvability}

Assume that $E \rightarrow X$ is a holomorphic vector bundle; this means that the transition matrices have entries in $\mathscr{O}_{X}$. For instance if we have a global embedding $i: X \rightarrow \Omega$ and a holomorphic vector bundle $F \rightarrow \Omega$, then $F$ defines a vector bundle $i^{*} F \rightarrow X$. The sheaves $\mathscr{A}_{X}^{*}(E)$ give rise to a fine resolution of the sheaf $\mathscr{O}_{X}(E)$, and by standard homological algebra we have the isomorphisms

$$
H^{q}(X, \mathscr{O}(E))=\frac{\operatorname{Ker}\left(\mathscr{A}^{q}(X, E) \stackrel{\bar{\partial}}{\rightarrow} \mathscr{A}^{q+1}(X, E)\right)}{\operatorname{Im}\left(\mathscr{A}^{q-1}(X, E) \stackrel{\bar{\partial}}{\rightarrow} \mathscr{A}^{q}(X, E)\right)}, \quad q \geq 1 .
$$

Thus, if $\phi \in \mathscr{A}^{q+1}(X, E), \bar{\partial} \phi=0$, and its canonical cohomology class vanishes, then the equation $\bar{\partial} \psi=\phi$ has a global solution in $\mathscr{A}^{q}(X, E)$. In particular, the equation 
is always solvable if $X$ is Stein. If for instance $X$ is a pure-dimensional projective variety $i: X \rightarrow \mathbb{P}^{N}$, then the $\bar{\partial}$-equation is solvable, e.g., if $E$ is a sufficiently ample line bundle.

\section{Locally complete intersections}

Let us consider the special case when $X$ locally is a complete intersection, i.e., given a local embedding $i: X \rightarrow \Omega \subset \mathbb{C}^{N}$ there are global sections $f_{j}$ of $\mathscr{O}\left(d_{j}\right) \rightarrow \mathbb{P}^{N}$ such that $\mathcal{J}=\left(f_{1}, \ldots, f_{p}\right)$, where $p=N-n$. In particular, $Z=\left\{f_{1}=\cdots=f_{p}=0\right\}$. In this case $\mathcal{H}$ om $\left(\mathscr{O}_{\Omega} / \mathcal{J}, \mathcal{C H}_{\Omega}\right)$ is generated by the single current

$$
\mu=\bar{\partial} \frac{1}{f_{p}} \wedge \cdots \wedge \bar{\partial} \frac{1}{f_{1}} \wedge d z_{1} \wedge \cdots \wedge d z_{N},
$$

see, e.g., [3]. Each smooth $(0, q)$-form $\phi$ in $\mathscr{E}_{X}^{0, q}$ is thus represented by a current $\Phi \wedge \mu$, where $\Phi$ is smooth in a neighborhood of $Z$ and $i^{*} \Phi=\phi$. Moreover, $X$ is CohenMacaulay so $X_{\text {reg }}$ coincides with the part of $X$ where $Z$ is regular, and $\bar{\partial} \phi=\psi$ if and only if $\bar{\partial}(\phi \wedge \mu)=\psi \wedge \mu$.

Henkin and Polyakov introduced, see [17, Definition 1.3], the notion of residual currents $\phi$ of bidegree $(0, q)$ on a locally complete intersection $X \subset \mathbb{P}^{N}$, and the $\bar{\partial}$-equation $\bar{\partial} \psi=\phi$. Their currents $\phi$ correspond to our $\phi$ in $\mathscr{E}_{X}^{0, q}$ and their $\bar{\partial}$-operator on such currents coincides with ours.

Remark 12.1 In [18] Henkin and Polyakov consider a global reduced complete intersection $X \subset \mathbb{P}^{N}$. They prove, by a global explicit formula, that if $\phi$ is a global $\bar{\partial}$-closed smooth $(0, q)$-form with values in $\mathscr{O}(\ell), \ell=d_{1}+\cdots d_{p}-N-1$, then there is a smooth solution to $\bar{\partial} \psi=\phi$ at least on $X_{\text {reg }}$, if $1 \leq q \leq n-1$. When $q=n$ a necessary obstruction term occurs. However, their meaning of " $\bar{\partial}$-closed" is that locally there is a representative $\Phi$ of $\phi$ and smooth $g_{j}$ such that $\bar{\partial} \Phi=g_{1} f_{1}+\cdots+g_{p} f_{p}$. If this holds, then clearly $\bar{\partial} \phi=0$. The converse implication is not true, see Example 12.2 below. It is not clear to us whether their formula gives a solution under the weaker assumption that $\bar{\partial} \phi=0$, neither do we know whether their solution admits some intrinsic extension across $X_{\text {sing }}$ as a current on $X$.

Example 12.2 Let $X=\{f=0\} \subset \Omega \subset \mathbb{C}^{n+1}$ be a reduced hypersurface, and assume that $d f \neq 0$ on $X_{\text {reg }}$, so that $\mathcal{J}=(f)$. Let $\phi$ be a smooth $(0, q)$-form in a neighborhood of some point $x$ on $X$ such that $\bar{\partial} \phi=0$. We claim that $\bar{\partial} u=\phi$ has a smooth solution $u$ if and only if $\phi$ has a smooth representative $\Phi$ in ambient space such that $\bar{\partial} \Phi=f g$ for some smooth form $g$. In fact, if such a $\Phi$ exists then $0=f \bar{\partial} g$ and thus $\bar{\partial} g=0$. Therefore, $g=\bar{\partial} \gamma$ for some smooth $\gamma$ (in a Stein neighborhood of $x$ in ambient space) and hence $\bar{\partial}(\Phi-f \gamma)=0$. Thus there is a smooth $U$ such that $\bar{\partial} U=\Phi-f \gamma$; this means that $u=i^{*} U$ is a smooth solution to $\bar{\partial} u=\phi$. Conversely, if $u$ is a smooth solution, then $u=i^{*} U$ for some smooth $U$ in ambient space, and thus $\Phi=\bar{\partial} U$ is a representative of $\phi$ in ambient space. Thus $\bar{\partial} \Phi=f g$ (with $g=0$ ). 
There are examples of hypersurfaces $X$ where there exist smooth $\phi$ with $\bar{\partial} \phi=0$ that do not admit smooth solutions to $\bar{\partial} u=\phi$, see, e.g., [6, Example 1.1]. It follows that such a $\phi$ cannot have a representative $\Phi$ in ambient space as above.

Acknowledgements We thank the referee for very careful reading and many valuable remarks.

Open Access This article is distributed under the terms of the Creative Commons Attribution 4.0 International License (http://creativecommons.org/licenses/by/4.0/), which permits unrestricted use, distribution, and reproduction in any medium, provided you give appropriate credit to the original author(s) and the source, provide a link to the Creative Commons license, and indicate if changes were made.

\section{References}

1. Andersson, M.: Uniqueness and factorization of Coleff-Herrera currents. Ann. Fac. Sci. Toulouse Math. 18(4), 651-661 (2009)

2. Andersson, M.: A residue criterion for strong holomorphicity. Ark. Mat. 48(1), 1-15 (2010)

3. Andersson, M.: Coleff-Herrera currents, duality, and Noetherian operators. Bull. Soc. Math. France 139, 535-554 (2011)

4. Andersson, M.: Pseudomeromorphic currents on subvarieties. Complex Var. Elliptic Equ. 61(11), 1533-1540 (2016)

5. Andersson, M., Lärkäng, R., Ruppenthal, J., Samuelsson Kalm, H., Wulcan, E.: Estimates for the $\bar{\partial}$-equation on canonical surfaces (2018). arXiv:1804.01004 [math.CV]

6. Andersson, M., Samuelsson, H.: A Dolbeault-Grothendieck lemma on complex spaces via Koppelman formulas. Invent. Math. 190, 261-297 (2012)

7. Andersson, M., Wulcan, E.: Residue currents with prescribed annihilator ideals. Ann. Sci. École Norm. Sup. 40, 985-1007 (2007)

8. Andersson, M., Wulcan, E.: Decomposition of residue currents. J. Reine Angew. Math. 638, 103-118 (2010)

9. Andersson, M., Wulcan, E.: Global effective versions of the Briançon-Skoda-Huneke theorem. Invent. Math 200(2), 607-651 (2015)

10. Andersson, M., Wulcan, E.: Direct images of semi-meromorphic currents. Ann. Inst. Fourier. arXiv:1411.4832v2 [math.CV]

11. Andersson, M., Wulcan, E.: Regularity of pseudomeromorphic currents (2017). arXiv:1703.01247 [math.CV]

12. Barlet D.: Le faisceau $\omega_{X}$ sur un espace analytique $X$ de dimension pure. In: Fonctions de plusieurs variables complexes, III (Sém. François Norguet, 1975-1977), Lecture Notes in Math., vol. 670, pp. 187-204. Springer, Berlin (1978)

13. Björk, J.-E.: Residues and mathcalD-modules. The legacy of Niels Henrik Abel, pp. 605-651. Springer, Berlin (2004)

14. Eisenbud, D.: Commutative algebra. With a view toward algebraic geometry. Graduate Texts in Mathematics, vol. 150. Springer, New-York (1995)

15. Fornæss, J.E., Øvrelid, N., Vassiliadou, S.: Semiglobal results for $\bar{\partial}$ on a complex space with arbitrary singularities. Proc. Am. Math. Soc. 133(8), 2377-2386 (2005)

16. Henkin, G., Passare, M.: Abelian differentials on singular varieties and variations on a theorem of Lie-Griffiths. Invent. Math. 135(2), 297-328 (1999)

17. Henkin, G., Polyakov, P.: Residual $\bar{\partial}$-cohomology and the complex Radon transform on subvarieties of $\mathbb{C} P^{n}$. Math. Ann. 354, 497-527 (2012)

18. Henkin, G., Polyakov, P.: Explicit Hodge-type decomposition on projective complete intersections. J. Geom. Anal. 26(1), 672-713 (2016)

19. Herrera, M., Lieberman, D.: Residues and principal values on complex spaces. Math. Ann. 194, 259294 (1971)

20. Hörmander, L.: The analysis of linear partial differential operators. I. Distribution theory and Fourier analysis. Grundlehren der Mathematischen Wissenschaften, vol. 256. Springer, Berlin (1983)

21. Lärkäng, R.: A comparison formula for residue currents. Math. Scand. arXiv:1207.1279 [math.CV]

22. Lärkäng, R.: Explicit versions of the local duality theorem in $\mathbb{C}^{n}$ (2015). arXiv:1510.01965 [math.CV] 
23. Lärkäng, R., Ruppenthal, J.: Koppelman formulas on affine cones over smooth projective complete intersections. Indiana Univ. Math. J. 67(2), 753-780 (2018)

24. Malgrange, B.: Sur les fonctions différentiables et les ensembles analytiques. Bull. Soc. Math. France 91, 113-127 (1963)

25. Malgrange, B.: Ideals of differentiable functions. Tata Institute of Fundamental Research Studies in Mathematics, No. 3, Tata Institute of Fundamental Research, Bombay (1967)

26. Narasimhan, R.: Introduction to the theory of analytic spaces. Lecture Notes in Mathematics, No. 25, Springer, Berlin (1966)

27. Øvrelid, N., Vassiliadou, S.: $L^{2}-\bar{\partial}$-cohomology groups of some singular complex spaces. Invent. Math. 192(2), 413-458 (2013)

28. Pardon, W., Stern, M.: $L^{2}-\bar{\partial}$-cohomology of complex projective varieties. J. Am. Math. Soc. 4(3), 603-621 (1991)

29. Pardon, W., Stern, M.: Pure Hodge structure on the $L^{2}$-cohomology of varieties with isolated singularities. J. Reine Angew. Math. 533, 55-80 (2001)

30. Roos, J.-E.: Bidualité et structure des foncteurs dérivés de lim dans la catégorie des modules sur un anneau régulier. C. R. Acad. Sci. Paris 254, 1720-1722 (1962)

31. Ruppenthal, J.: $L^{2}$-theory for the $\bar{\partial}$-operator on compact complex spaces. Duke Math. J. 163(15), 2887-2934 (2014)

32. Sznajdman, J.: A Briançon-Skoda type result for a non-reduced analytic space. J. Reine Angew. Math. arXiv:1001.0322 [math.CV]

33. Tsikh, A.K.: Multidimensional residues and their applications. Translations of Mathematical Monographs, vol. 103. American Mathematical Society, Providence (1992) 